\title{
SYNTHESIS AND ANTI-INFLAMMATORY ACTIVITY OF SOME FUSED PYRIMIDINES
}

Nehad A. El-Sayed ${ }^{1}$, Awatef E. Farag ${ }^{1}$, Mohamed T. El-Saadi ${ }^{2}$, Samir $^{2}$ M. El-Moghazy ${ }^{1}$ and Hekmat A. Abdel- Latif ${ }^{3}$

${ }^{1}$ Department of Pharmaceutical Chemistry, Faculty of Pharmacy, Cairo University

${ }^{2}$ Department of Pharmaceutical Chemistry, Faculty of Pharmacy, Beni Sueif University

${ }^{3}$ Department of Pharmacology, Faculty of Pharmacy, Cairo University

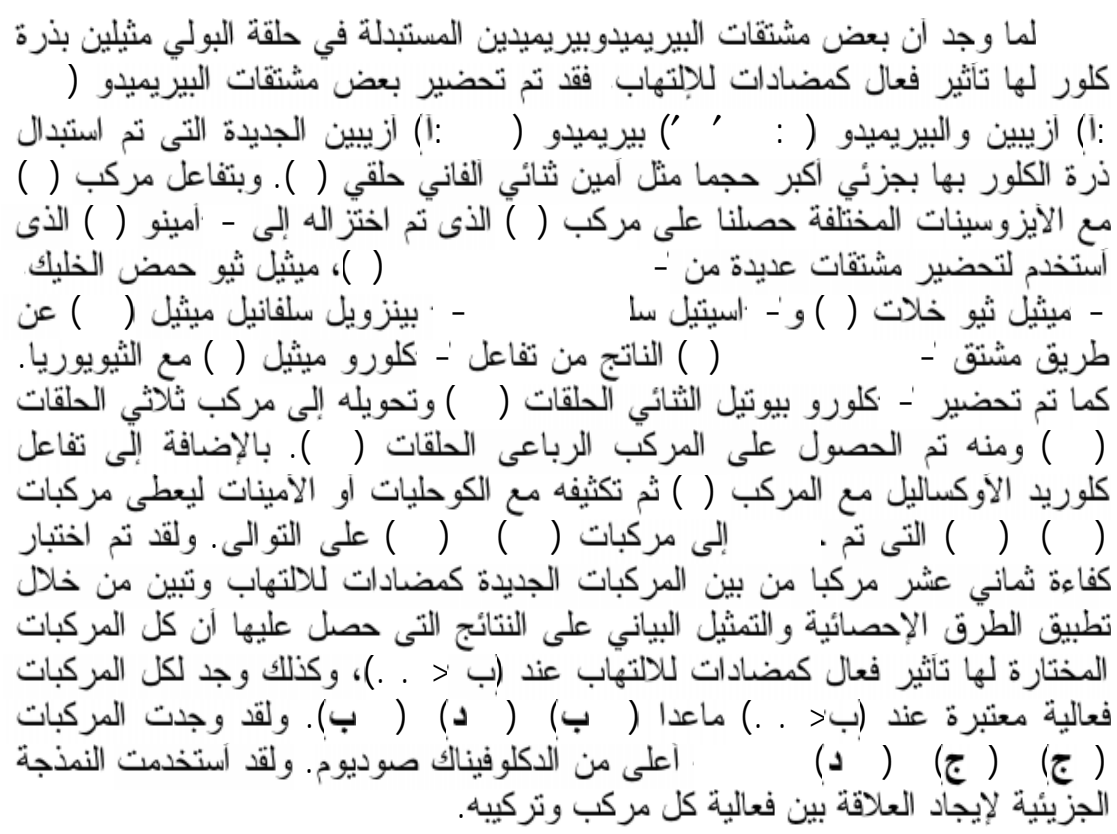

Seeking new anti-inflammatory agents and based on molecular modeling studies, design and synthesis of pyrimidoazepine and pyrimidopyrimidoazepine derivatives substituted in the polymethylene ring with different alicyclic secondary amines was performed. Thus, reacting 2-dicyanomethylidenoperhydroazepine 1 with sulphuryl chloride furnished the 6-chloro derivative 2.The oiminonitriles $\mathbf{3}$ were obtained via the reaction of $\mathbf{2}$ with isopropyl or

Received in 13/9/2006 \& Accepted in 26/12/2006

*Corresponding author E-mail address: Nehad_El_sayed@yahoo.com 
phenyl isocyanate. Refluxing 3 with morpholine or piperidine afforded the 5-morpholino or piperidino 3-imino derivatives 4. Reduction of the latter compounds furnished the corresponding 3amino derivatives 5. Preparation of the 3-chloromethyl pyrimidoazepine derivatives $\mathbf{6}$ was achieved via the reaction of $\mathbf{5}$ with chloroacetyl chloride. Reacting 6 with thiourea and further decomposition of the methyl isothiourea salts gave the 3-thiomethyl derivatives 7. Preparation of thioethers 8 and 9 was done through the reaction of 7 with methyl iodide, chloroacetic acid or ethyl chloroacetate. Refluxing the 3-thiomethyl compounds 7 with acetyl or benzoyl chloride yielded the 3-thioester compounds 10. The uncyclized (4-chlorobutanamide) derivatives 11 were obtained through the reaction of the enaminonitriles 5 with chlorobutyryl chloride. Refluxing compounds 11 with alcoholic hydrochloric acid solution yielded the 3-chloropropyl pyrimidopyrimidoazepine derivatives 12.These tricycles when reacted with different primary or secondary amines yielded the tetracyclic ring system pyrrolopyrimidopyrimidoazepines 13. Reacting 5 with oxalyl chloride gave the 3-chlorocarbonyl derivatives, which were reacted without separation with different alcohols and amines affording the corresponding 3-carbamoylformate 14 and 1 - $N$-substituted -2oxoacetamide 15, respectively. Intramolecular cyclization of these latter compounds yielded their tricyclic counterparts 16 and 17, respectively. Eighteen representative compounds were screened for their anti-inflammatory activity using diclofenac as reference drug. Also, molecular modelling was performed.

\section{INTRODUCTION}

COX-inhibitors (antiinflammatory and antipyretic drugs) are widely used classes of therapeutic agents. Previous studies done by Ebeid et al on the nitrogen bridgehead condensed pyrimidine derivatives led to the discovery of new active antiinflammatory compounds belonging to pyrimido[1,6-a]azepines $\mathbf{I}^{1}$ and pyrimido[4,5:4,5]pyrimido[1,6-a]azepines II ring system. ${ }^{1}$ Moreover, further studies on several pyrimido $[4,5: 4,5]$ pyrimido[ $[1,6$-a]azepine deri- vatives revealed that introduction of chlorine atom in the polymethylene ring at position 12 affording compound III $^{2}$ resulted in significant improvement in anti-inflammatory activity.<smiles>C=CCn1c(=N)c(C#N)c2n(c1=S)CCCCC2</smiles>

I 
<smiles>CCCCCCN1C(=O)N2CCCCC(Cl)C2C2=C1N=C(CCl)NC2</smiles>

Based on these findings and for further exploration of the pyrimidopyrimidoazepine ring system in the field of anti-inflammatory agents, molecular modeling calculations have been carried out on compounds II and III in order to correlate the spatial changes of the molecule with its activity.

It was observed that twisting increases between the polymethylene ring and the rest of the molecule by about $15^{\circ}$ upon insertion of the chlorine atom, leading to increased activity (Table I).

Moreover, a slight decrease in torsion angle $\mathrm{H}(\mathrm{Cl})(\mathrm{N})-12-12 \mathrm{a}-12 \mathrm{~b}$ was perceived, with retention of the activity in the significant range, when a bulky moiety such as a alicyclic secondary amine was substituted for the chlorine atom.

It was of interest to extrapolate this concept to the bicyclic pyrimido[1,6-a]azepine derivative I substituted by an alicyclic secondary amine in position 5 affording compound $\mathbf{4 c}$ where this modification was accompanied by a significant increase in twisting of torsion angle and activity (Table II).

On the light of these findings and as a part of ongoing search for new potent anti-inflammatory agents, this work pertains to the design and synthesis of new series of pyrimido [1,6-a]azepine IV and V, and pyrimido[4',5':4,5]pyrimido[1,6-a]azepine derivatives VI substituted in the polymethylene ring with piperidine or morpholine moiety in order to investigate the influence of the conformational changes of the studied pharmacophores on the antiinflammatory activity.<smiles>[R7]c1nc2c(c(=O)[nH]1)C1C([R8])CCCCN1C(=O)N2[R]=[W]</smiles>

In the same vein, the research aims to investigate the influence of increasing the bulkiness of the molecule on the twisting of torsion angles $12-12 \mathrm{a}-12 \mathrm{~b}-(\mathrm{CO})$ and $\mathrm{Cl}(\mathrm{N})$ $12-12 \mathrm{a}-12 \mathrm{~b}$, as well as on the antiinflammatory activity. Consequently, annulation of an additional ring on the tricyclic pyrimido[4',5':4,5] pyrimido[1,6-a]azepine ring system VI was done yielding compound VII.<smiles>O=C1N(P)c2nc3n4c(=O)c2C(CCC4)C(Br)CCCCN13</smiles> 
Table I: Torsion angles of atoms $5-4 \mathrm{a}-4-\mathrm{C}(\mathrm{CN})$ and $\mathrm{H}(\mathrm{N}) 5-4 \mathrm{a}-4$ and percentage activity.

\begin{tabular}{|c|c|c|c||}
\hline $\begin{array}{c}\text { Compound } \\
\text { No. }\end{array}$ & $\begin{array}{c}\text { Torsion angle of } \\
\text { Atoms } \\
\text { 5-4a-4-C(CN) }\end{array}$ & $\begin{array}{c}\text { Torsion angle of } \\
\text { atoms } \\
\text { ( }\end{array}$ & $\begin{array}{c}\text { Activity } 5-4 a-4 \\
\%\end{array}$ \\
\hline I & 3.0353 & $\begin{array}{c}46.2324 \\
(-67.3853)\end{array}$ & 23 \\
\hline $\mathbf{4 c}$ & -7.9882 & -88.6645 & 88 \\
\hline $\mathbf{5 c}$ & 3.4387 & -58.8528 & 70 \\
\hline $\mathbf{1 4 c}$ & 2.7157 & -57.5781 & 84.2 \\
\hline $\mathbf{1 4 d}$ & 3.4157 & -56.5781 & 81 \\
\hline $\mathbf{1 4 e}$ & 4.0708 & -58.0348 & 60 \\
\hline $\mathbf{1 5 c}$ & 5.5007 & -58.5448 & 64 \\
\hline $\mathbf{1 5 d}$ & 6.3661 & -59.3181 & 55.7 \\
\hline $\mathbf{1 5}$ & 6.1151 & -58.9613 & 46.4 \\
\hline
\end{tabular}

Table II: Torsion angles of atoms $12-12 \mathrm{a}-12 \mathrm{~b}-\mathrm{CO}$ and $\mathrm{Cl}(\mathrm{N})-12-12 \mathrm{a}-12 \mathrm{~b}$ and percentage activity.

\begin{tabular}{||c|c|c|c||}
\hline $\begin{array}{c}\text { Compound } \\
\text { No. }\end{array}$ & $\begin{array}{c}\text { Torsion angle }^{\circ} \\
12-12 \mathrm{a}-12 \mathrm{~b}-\mathrm{CO}\end{array}$ & $\begin{array}{c}\text { Torsion angle }^{\circ} \\
\text { Cl(N)-12-12a-12b }\end{array}$ & $\begin{array}{c}\text { Activity } \\
\%\end{array}$ \\
\hline II & 72.3555 & $\begin{array}{c}48.3943 \\
(-66.5016)\end{array}$ & 38 \\
\hline III & 87.3819 & -63.7448 & 76 \\
\hline $\mathbf{6 c}$ & 89.6907 & -52.0906 & 60 \\
\hline $\mathbf{7 c}$ & 90.2238 & -43.8014 & 57.1 \\
\hline $\mathbf{8 c}$ & 88.9228 & -43.1943 & 81.4 \\
\hline $\mathbf{1 0 b}$ & 89.4528 & -51.7408 & 74.2 \\
\hline $\mathbf{1 0 c}$ & 89.8137 & -52.8788 & 71.4 \\
\hline $\mathbf{1 2 b}$ & 89.8111 & 54.6651 & 53.5 \\
\hline $\mathbf{1 3 b}$ & 91.4670 & -49.9437 & 41.4 \\
\hline $\mathbf{1 6 b}$ & 89.2502 & -54.4143 & 49.2 \\
\hline $\mathbf{1 7 a}$ & 84.1508 & -52.1845 & 55.7 \\
\hline
\end{tabular}

\section{MATERIALS AND METHODS}

Melting points ( ${ }^{\circ}$, uncorrected) were recorded on an Electrothermal 1 A 9100 Digital Melting Point Apparatus. IR spectra as $\mathrm{KBr}$ disk were recorded on a Bruker Vector 22 Germany. ${ }^{1} \mathrm{H}-\mathrm{NMR}$ spectra were recorded in DMSO-d $\mathrm{d}_{6}$ or $\mathrm{CDCl}_{3}$ on Jeol FT 90Q, $300 \mathrm{MHz}$ using TMS as internal standard. Mass spectra were performed on Fennigan MAT, SSQ 7000, Mass Spectrometer with Electron Impact at $70 \mathrm{eV}$. Elemental analyses were carried out at the Microanalytical Center, Faculty of Science, Cairo University. TLC was performed in chloroform using TLC aluminium sheet protected with silica gel 60 F254- layer thickness $0.2 \mathrm{~mm}$. 
Synthesis of compounds

2-Dicyanomethylidenoperhydroazepine $\quad 1,3$ 3-chloro-2-dicyanomethylidenoperhydroazepine $\mathbf{2}^{2}$ and 5-chloro-3-imino-2-isopropyl or phenyl-1,2,3,5,6,7,8,9-octahydro-1oxopyrimido[1,6-a]azepine-4-carbonitrile $\mathbf{3}^{2}$ were prepared according to the reported methods.

3-Imino-2-isopropyl- or phenyl-5morpholino- or piperidino-1-oxo1,2,3,5,6,7,8,9-octahydropyrimido[1,6-a]azepine-4-carbonitrile (4a-c)

A mixture of 3a,b $(10 \mathrm{mmol})$ and morpholine or piperidine $(15 \mathrm{mmol})$ in absolute ethanol $(10 \mathrm{ml})$ was heated under reflux for 6 hours and evaporated under vacuum to dryness. The remaining residue was triturated with ether; the separated crystals were filtered and recrystallized from aqueous ethanol (Table III). 4a: IR (KBr) $3225(\mathrm{NH}), 2217(\mathrm{CN}), 1595$ (NCON). 4b: IR (KBr) 3277, 2217, 1600. 4c: IR (KBr) 3247, 2225, 1630. 4a: ${ }^{1} \mathrm{H}-\mathrm{NMR}\left(\mathrm{CDCl}_{3}\right) \delta=1.31-1.39$ (m, C-6 $\left.\left(\mathrm{H}_{2}\right), \mathrm{C}-7\left(\mathrm{H}_{2}\right), 4 \mathrm{H}\right) ; 1.85$ (d, $\left.\mathrm{CH}\left(\underline{\mathrm{CH}}_{3}\right)_{2}, 6 \mathrm{H}\right) ; 1.96-2.21$ (m, C$\left.8\left(\mathrm{H}_{2}\right), 2 \mathrm{C}-\mathrm{b}\left(\mathrm{H}_{2}\right), \mathrm{C}-\mathrm{c}\left(\mathrm{H}_{2}\right), 8 \mathrm{H}\right) ; 2.55$ $\left(\mathrm{t}, 2 \mathrm{C}-\mathrm{a}\left(\mathrm{H}_{2}\right), 4 \mathrm{H}\right) ; 3.53\left(\mathrm{t}, \mathrm{C}-9\left(\mathrm{H}_{2}\right)\right.$, $2 \mathrm{H}) ; 4.21$ (septet, $\left.\underline{\mathrm{CH}}\left(\mathrm{CH}_{3}\right)_{2}, 1 \mathrm{H}\right)$; $4.73(\mathrm{t}, \mathrm{C}-5(\mathrm{H}), 1 \mathrm{H}) ; 8.91$ (s, NH, $1 \mathrm{H})$. 4c: ${ }^{1} \mathrm{H}-\mathrm{NMR}\left(\mathrm{CDCl}_{3}\right) \delta=1.18-$ 1.35 (m, C-6 $\left.\left(\mathrm{H}_{2}\right), \mathrm{C}-7\left(\mathrm{H}_{2}\right), 4 \mathrm{H}\right)$; 1.96$2.21\left(\mathrm{~m}, \mathrm{C}-8\left(\mathrm{H}_{2}\right), 2 \mathrm{H}\right) ; 2.49(\mathrm{t}, 2 \mathrm{C}-$ $\left.\mathrm{a}\left(\mathrm{H}_{2}\right), 4 \mathrm{H}\right) ; 3.13\left(\mathrm{t}, \mathrm{C}-9\left(\mathrm{H}_{2}\right), 2 \mathrm{H}\right)$; $3.71\left(\mathrm{t}, \mathrm{C}-5(\mathrm{H}), 2 \mathrm{C}-\mathrm{b}\left(\mathrm{H}_{2}\right), 5 \mathrm{H}\right)$; 7.17$7.41\left(\mathrm{~m}, \mathrm{C}_{6} \mathrm{H}_{5}, 5 \mathrm{H}\right) ; 9.41$ (s, NH, $\left.1 \mathrm{H}\right)$. 4a: $\mathrm{MS}: \mathrm{m} / \mathrm{z}(\%)=\mathrm{M}^{+} 329$ (0.08), 133 (100).
3-Amino-2-isopropyl- or phenyl-5morpholino- or piperidino-1-oxo1,2,4a,5,6,7,8,9-octahydropyrimido [1,6-a]azepine-4-carbonitrile (5a-c)

To a stirred mixture of 4a-c (10 mmol) in absolute ethanol $(20 \mathrm{ml})$, sodium borohydride $(0.2 \mathrm{~g}, 5 \mathrm{mmol})$ was added in portions and stirring was continued for one hour. The reaction mixture was left to stand overnight at room temperature; the separated crystals were filtered, washed with water and recrystallized from aqueous ethanol (Table III) .5a: IR (KBr) 3280, $3166\left(\mathrm{NH}_{2}\right), 2217$ $(\mathrm{CN}), 1620(\mathrm{~N}-\mathrm{CO}-\mathrm{N}) .5 \mathrm{~b}: \mathrm{IR}(\mathrm{KBr})$ 3210, 3123, 2220, 1645. 5c: IR (KBr) 3277, 3260, 2215, 1600. 5a: ${ }^{1} \mathrm{H}-\mathrm{NMR}$ $\left(\right.$ DMSO $\left.^{-} \mathrm{d}_{6}\right): \delta=1.31-1.39 \quad(\mathrm{~m}, \mathrm{C}-$ $\left.6\left(\mathrm{H}_{2}\right), \quad \mathrm{C}-7\left(\mathrm{H}_{2}\right), \quad 4 \mathrm{H}\right) ; \quad 1.85 \quad(\mathrm{~d}$, $\left.\mathrm{CH}\left(\mathrm{CH}_{3}\right)_{2}, 6 \mathrm{H}\right) ; 1.96-2.21 \quad(\mathrm{~m}, \mathrm{C}-$ $\left.8\left(\mathrm{H}_{2}\right), 2 \mathrm{C}-\mathrm{b}\left(\mathrm{H}_{2}\right), \mathrm{C}-\mathrm{c}\left(\mathrm{H}_{2}\right), 8 \mathrm{H}\right) ; 2.51(\mathrm{t}$, $\left.2 \mathrm{C}-\mathrm{a}\left(\mathrm{H}_{2}\right), 4 \mathrm{H}\right) ; 3.75\left(\mathrm{t}, \mathrm{C}-9\left(\mathrm{H}_{2}\right), 2 \mathrm{H}\right)$; 4.35 (septet, $\left.\underline{\mathrm{CH}}\left(\mathrm{CH}_{3}\right)_{2}, 1 \mathrm{H}\right) ; 4.73$ (d, $\mathrm{C}-4 \mathrm{a}(\mathrm{H}), 1 \mathrm{H}) ; 5.13$ (q, C-5(H), 1H); 8.53 (s, $\left.\mathrm{NH}_{2}, \quad 2 \mathrm{H}\right)$. 5c: ${ }^{1} \mathrm{H}-\mathrm{NMR}$ $\left(\right.$ DMSO $\left._{6}\right): \delta=1.29-1.46 \quad(\mathrm{~m}, \mathrm{C}-$ $\left.6\left(\mathrm{H}_{2}\right), \mathrm{C}-7\left(\mathrm{H}_{2}\right), 4 \mathrm{H}\right) ; 1.86-2.22(\mathrm{~m}, \mathrm{C}-$ $\left.8\left(\mathrm{H}_{2}\right), 2 \mathrm{H}\right) ; 2.33\left(\mathrm{t}, 2 \mathrm{C}-\mathrm{a}\left(\mathrm{H}_{2}\right), 4 \mathrm{H}\right)$; $3.20\left(\mathrm{t}, \mathrm{C}-9\left(\mathrm{H}_{2}\right), 2 \mathrm{H}\right) ; 3.79(\mathrm{t}, 2 \mathrm{C}-$ $\left.\mathrm{b}\left(\mathrm{H}_{2}\right), 4 \mathrm{H}\right) ; 4.56(\mathrm{q}, \mathrm{C}-5(\mathrm{H}), 1 \mathrm{H})$; $4.80(\mathrm{~d}, \mathrm{C}-4 \mathrm{a}(\mathrm{H}), 1 \mathrm{H}) ; 6.75-7.26(\mathrm{~m}$, $\left.\mathrm{C}_{6} \mathrm{H}_{5}, 5 \mathrm{H}\right) ; 9.51\left(\mathrm{~s}, \mathrm{NH}_{2}, 2 \mathrm{H}\right) .5 \mathrm{a}: \mathrm{MS}$ : $\mathrm{m} / \mathrm{z}(\%)=\mathrm{M}^{+} 331$ (18), 210 (100).

3-Chloromethyl-5-isopropyl- or phenyl-12-morpholino- or piperi-

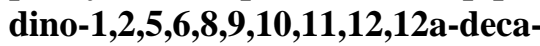
hydropyrimido $\left[4^{\prime}, 5^{\prime}: 4,5\right]$ pyrimido [1,6-a]azepine-1,6-dione (6a-c)

A mixture of 5a-c $(10 \mathrm{mmol})$, chloroacetyl chloride $(15 \mathrm{mmol})$ and triethylamine $(2.5 \mathrm{ml})$ in dry benzene 
Table III: Physical and analytical data of the prepared compounds 4a-c, 5a-c, 6a-c and 7a-c.

\begin{tabular}{|c|c|c|c|c|c|c|c|}
\hline \multirow{2}{*}{ No } & \multirow{2}{*}{$\mathrm{R}$} & \multirow{2}{*}{$\mathrm{R}^{1}$} & \multirow{2}{*}{$\begin{array}{l}\text { Yield \% } \\
\text { M.P }^{\circ}\end{array}$} & \multirow{2}{*}{$\begin{array}{l}\text { Mol. formula } \\
\text { (M.wt.) }\end{array}$} & \multicolumn{3}{|c|}{ Microanalytical data } \\
\hline & & & & & & Calcd. & Found \\
\hline $4 \mathbf{a}$ & $\mathrm{CH}\left(\mathrm{CH}_{3}\right)_{2}$ & & $\begin{array}{c}70 \\
200-4\end{array}$ & $\begin{array}{c}\mathrm{C}_{18} \mathrm{H}_{27} \mathrm{~N}_{5} \mathrm{O} \\
329.44\end{array}$ & $\begin{array}{l}\mathrm{C} \\
\mathrm{H} \\
\mathrm{N}\end{array}$ & $\begin{array}{c}65.62 \\
8.26 \\
21.26\end{array}$ & $\begin{array}{c}65.91 \\
8.53 \\
21.21\end{array}$ \\
\hline b & $\mathrm{C}_{6} \mathrm{H}_{5}$ & & $\begin{array}{c}65 \\
170-3\end{array}$ & $\begin{array}{c}\mathrm{C}_{21} \mathrm{H}_{25} \mathrm{~N}_{5} \mathrm{O} \\
363.46\end{array}$ & $\begin{array}{l}\mathrm{C} \\
\mathrm{H} \\
\mathrm{N} \\
\end{array}$ & $\begin{array}{c}69.40 \\
6.93 \\
19.27 \\
\end{array}$ & $\begin{array}{c}69.25 \\
6.34 \\
19.56 \\
\end{array}$ \\
\hline c & $\mathrm{C}_{6} \mathrm{H}_{5}$ & & $\begin{array}{c}80 \\
155-7\end{array}$ & $\begin{array}{c}\mathrm{C}_{20} \mathrm{H}_{23} \mathrm{~N}_{5} \mathrm{O}_{2} \\
365.43\end{array}$ & $\begin{array}{l}\mathrm{C} \\
\mathrm{H} \\
\mathrm{N}\end{array}$ & $\begin{array}{c}65.73 \\
6.34 \\
19.16 \\
\end{array}$ & $\begin{array}{c}65.23 \\
6.45 \\
19.42 \\
\end{array}$ \\
\hline $5 \mathbf{a}$ & $\mathrm{CH}\left(\mathrm{CH}_{3}\right)_{2}$ & & $\begin{array}{c}60 \\
167-9\end{array}$ & $\begin{array}{c}\mathrm{C}_{18} \mathrm{H}_{29} \mathrm{~N}_{5} \mathrm{O} \\
331.46\end{array}$ & $\begin{array}{l}\mathrm{C} \\
\mathrm{H} \\
\mathrm{N} \\
\end{array}$ & $\begin{array}{c}65.23 \\
8.82 \\
21.13 \\
\end{array}$ & $\begin{array}{c}64.75 \\
8.32 \\
21.14 \\
\end{array}$ \\
\hline b & $\mathrm{C}_{6} \mathrm{H}_{5}$ & & $\begin{array}{c}70 \\
180-3\end{array}$ & $\begin{array}{c}\mathrm{C}_{21} \mathrm{H}_{27} \mathrm{~N}_{5} \mathrm{O} \\
365.47\end{array}$ & $\begin{array}{l}\mathrm{C} \\
\mathrm{H} \\
\mathrm{N} \\
\end{array}$ & $\begin{array}{c}69.01 \\
7.45 \\
19.16 \\
\end{array}$ & $\begin{array}{c}68.71 \\
6.95 \\
19.66 \\
\end{array}$ \\
\hline c & $\mathrm{C}_{6} \mathrm{H}_{5}$ & & $\begin{array}{c}85 \\
197-9\end{array}$ & $\begin{array}{c}\mathrm{C}_{20} \mathrm{H}_{25} \mathrm{~N}_{5} \mathrm{O}_{2} \\
367.46\end{array}$ & $\begin{array}{l}\mathrm{C} \\
\mathrm{H} \\
\mathrm{N}\end{array}$ & $\begin{array}{c}65.37 \\
6.86 \\
19.06\end{array}$ & $\begin{array}{c}65.10 \\
6.62 \\
19.57\end{array}$ \\
\hline $6 \mathbf{a}$ & $\mathrm{CH}\left(\mathrm{CH}_{3}\right)_{2}$ & & $\begin{array}{c}65 \\
170-3\end{array}$ & $\begin{array}{c}\mathrm{C}_{20} \mathrm{H}_{30} \mathrm{ClN}_{5} \mathrm{O}_{2} \\
407.94\end{array}$ & $\begin{array}{l}\mathrm{C} \\
\mathrm{H} \\
\mathrm{N}\end{array}$ & $\begin{array}{c}58.89 \\
7.41 \\
17.17\end{array}$ & $\begin{array}{c}58.55 \\
7.66 \\
16.72\end{array}$ \\
\hline b & $\mathrm{C}_{6} \mathrm{H}_{5}$ & & $\begin{array}{c}70 \\
200-4\end{array}$ & $\begin{array}{c}\mathrm{C}_{23} \mathrm{H}_{28} \mathrm{ClN}_{5} \mathrm{O}_{2} \\
441.96\end{array}$ & $\begin{array}{l}\mathrm{C} \\
\mathrm{H} \\
\mathrm{N}\end{array}$ & $\begin{array}{c}62.51 \\
6.39 \\
15.85\end{array}$ & $\begin{array}{c}62.72 \\
5.95 \\
15.43\end{array}$ \\
\hline c & $\mathrm{C}_{6} \mathrm{H}_{5}$ & & $\begin{array}{c}85 \\
183-5\end{array}$ & $\begin{array}{c}\mathrm{C}_{22} \mathrm{H}_{26} \mathrm{Cl} \mathrm{N}_{5} \mathrm{O}_{3} \\
443.93\end{array}$ & $\begin{array}{l}\mathrm{C} \\
\mathrm{H} \\
\mathrm{N}\end{array}$ & $\begin{array}{c}59.52 \\
5.90 \\
15.78\end{array}$ & $\begin{array}{c}59.41 \\
5.83 \\
16.15\end{array}$ \\
\hline $7 \mathbf{a}$ & $\mathrm{CH}\left(\mathrm{CH}_{3}\right)_{2}$ & & $\begin{array}{c}60 \\
200-3\end{array}$ & $\begin{array}{c}\mathrm{C}_{20} \mathrm{H}_{31} \mathrm{~N}_{5} \mathrm{O}_{2} \mathrm{~S} \\
405.56\end{array}$ & $\begin{array}{l}\mathrm{C} \\
\mathrm{H} \\
\mathrm{N} \\
\end{array}$ & $\begin{array}{c}59.23 \\
7.70 \\
17.27 \\
\end{array}$ & $\begin{array}{c}58.81 \\
7.83 \\
16.72 \\
\end{array}$ \\
\hline b & $\mathrm{C}_{6} \mathrm{H}_{5}$ & & $\begin{array}{c}75 \\
187-9\end{array}$ & $\begin{array}{c}\mathrm{C}_{23} \mathrm{H}_{29} \mathrm{~N}_{5} \mathrm{O}_{2} \mathrm{~S} \\
439.58\end{array}$ & $\begin{array}{l}\mathrm{C} \\
\mathrm{H} \\
\mathrm{N}\end{array}$ & $\begin{array}{c}62.84 \\
6.65 \\
15.93\end{array}$ & $\begin{array}{c}62.44 \\
6.31 \\
15.82 \\
\end{array}$ \\
\hline c & $\mathrm{C}_{6} \mathrm{H}_{5}$ & & $\begin{array}{c}80 \\
195-7\end{array}$ & $\begin{array}{c}\mathrm{C}_{22} \mathrm{H}_{27} \mathrm{~N}_{5} \mathrm{O}_{3} \mathrm{~S} \\
441.55\end{array}$ & $\begin{array}{l}\mathrm{C} \\
\mathrm{H} \\
\mathrm{N}\end{array}$ & $\begin{array}{c}59.84 \\
6.16 \\
15.84\end{array}$ & $\begin{array}{c}60.21 \\
5.62 \\
15.35\end{array}$ \\
\hline
\end{tabular}


$(10 \mathrm{ml})$ was refluxed at a temperature not exceeding $70^{\circ}$ for two hours and evaporated to dryness, under vacuum. The residue was triturated with petroleum ether; the separated crystals were filtered and recrystallized from aqueous ethanol, (Table III). 6a: IR (KBr) 3259, 1650 1595. 6b: IR (KBr) 3320, 1645, 1599. 6c: IR (KBr) 3326, 1648, 1596. 6a: ${ }^{1} \mathrm{H}-\mathrm{NMR}\left(\mathrm{CDCl}_{3}\right) \delta=1.31-1.39(\mathrm{~m}$, $\left.\mathrm{C}-10\left(\mathrm{H}_{2}\right), \mathrm{C}-11\left(\mathrm{H}_{2}\right), 4 \mathrm{H}\right) ; 1.85$ (d, $\left.\mathrm{CH}\left(\mathrm{CH}_{3}\right)_{2}, 6 \mathrm{H}\right) ; 1.96-2.21 \quad$ (m, C$\left.9\left(\mathrm{H}_{2}\right), 2 \mathrm{C}-\mathrm{b}\left(\mathrm{H}_{2}\right), \mathrm{C}-\mathrm{c}\left(\mathrm{H}_{2}\right), 8 \mathrm{H}\right) ; 2.95$ (t, $\left.2 \mathrm{C}-\mathrm{a}\left(\mathrm{H}_{2}\right), \quad 4 \mathrm{H}\right) ; \quad 3.33 \quad$ (septet, $\left.\underline{\mathrm{CH}}\left(\mathrm{CH}_{3}\right)_{2}, 1 \mathrm{H}\right) ; 3.60$ (s, $\left.\underline{\mathrm{CH}}_{2} \mathrm{Cl}, 2 \mathrm{H}\right)$; $4.13\left(\mathrm{t}, \mathrm{C}-8\left(\mathrm{H}_{2}\right), 2 \mathrm{H}\right) ; 5.13$ (d, C$12 \mathrm{a}(\mathrm{H}), 1 \mathrm{H}) ; 5.36(\mathrm{q}, \mathrm{C}-12(\mathrm{H}), 1 \mathrm{H})$; 8.53 (s, NH, 1H). 6b: ${ }^{1} \mathrm{H}-\mathrm{NMR}$ $\left(\mathrm{CDCl}_{3}\right) \delta=1.01-1.20\left(\mathrm{~m}, \mathrm{C}-10\left(\mathrm{H}_{2}\right)\right.$, $\left.\mathrm{C}-11\left(\mathrm{H}_{2}\right), \quad 4 \mathrm{H}\right) ; \quad 1.82-2.21 \quad(\mathrm{~m}, \mathrm{C}-$ $\left.9\left(\mathrm{H}_{2}\right), 2 \mathrm{C}-\mathrm{b}\left(\mathrm{H}_{2}\right), \mathrm{C}-\mathrm{c}\left(\mathrm{H}_{2}\right), 8 \mathrm{H}\right) ; 2.38$ $\left(\mathrm{t}, 2 \mathrm{C}-\mathrm{a}\left(\mathrm{H}_{2}\right), 4 \mathrm{H}\right) ; 3.73\left(\mathrm{t}, \mathrm{C}-8\left(\mathrm{H}_{2}\right)\right.$, $2 \mathrm{H}) ; 3.99$ (s, $\left.\mathrm{CH}_{2} \mathrm{Cl}, 2 \mathrm{H}\right) ; 4.25$ (q, C$12(\mathrm{H}), 1 \mathrm{H}) ; 5.19(\mathrm{~d}, \mathrm{C} .12 \mathrm{a}(\mathrm{H}), 1 \mathrm{H})$; 7.16-7.66 (m, $\left.\mathrm{C}_{6} \mathrm{H}_{5}, 5 \mathrm{H}\right) ; 8.54$ (s, NH, 1H). 6a: MS: $m / z(\%)=M^{+}+2409$ (0.05); $\mathrm{M}^{+} 407$ (8.22), 133 (100).

5-Isopropyl- or phenyl-12-morpholino- or piperidino-3-sulfanylmethyl-1,2,5,6,8,9,10,11,12,12adecahydropyrimido[4,5:4,5]pyrim ido[1,6-a]azepine-1,6-dione (7a-c)

A mixture of $\mathbf{6 a - c}(10 \mathrm{mmol})$ and thiourea $(0.76 \mathrm{~g}, 10 \mathrm{mmol})$ in ethanol $(10 \mathrm{ml})$ was heated under reflux for about three hours then left to cool. The separated crystals were filtered, dissolved in ice cooled solution of $10 \mathrm{NaOH}$ and filtered; the alkaline filtrate was acidified with dilute hydrochloric acid to $\mathrm{pH}$ 4. The formed precipitate was filtered, washed with water and crystallized from aqueous ethanol (Table III). 7a: IR (KBr) 3212, 1665, 1612. 7b: IR (KBr) 3310, 1653, 1615. 7c: IR (KBr) 3364, 1665, 1630. 7a: ${ }^{1} \mathrm{H}-\mathrm{NMR}$ $\left(\mathrm{CDCl}_{3}\right) \delta=1.31-1.39$ (m, C-10 $\left(\mathrm{H}_{2}\right)$, $\left.\mathrm{C}-11\left(\mathrm{H}_{2}\right), 4 \mathrm{H}\right) ; 1.45$ (d, $\mathrm{CH}\left(\mathrm{CH}_{3}\right)_{2}$, $6 \mathrm{H}) ; 1.59$ (s, SH, 1H); 1.96-2.21 (m, C-9 $\left.\left(\mathrm{H}_{2}\right), 2 \mathrm{C}-\mathrm{b}\left(\mathrm{H}_{2}\right), \mathrm{C}-\mathrm{c}\left(\mathrm{H}_{2}\right), 8 \mathrm{H}\right) ; 2.53$ (s, $\left.\mathrm{CH}_{2} \mathrm{SH}, 2 \mathrm{H}\right) ; 2.55\left(\mathrm{t}, 2 \mathrm{C}-\mathrm{a}\left(\mathrm{H}_{2}\right)\right.$, $4 \mathrm{H}) ; 3.73\left(\mathrm{t}, \quad \mathrm{C}-8\left(\mathrm{H}_{2}\right), 2 \mathrm{H}\right) ; 4.35$ (septet, $\left.\underline{\mathrm{CH}}\left(\mathrm{CH}_{3}\right)_{2}, 1 \mathrm{H}\right) ; 4.73$ (q, C. $12(\mathrm{H}), 1 \mathrm{H}) ; 5.21(\mathrm{~d}, \mathrm{C}-12 \mathrm{a}(\mathrm{H}), 1 \mathrm{H})$; 8.53 (s, NH, 1H). 7c: ${ }^{1} \mathrm{H}-\mathrm{NMR}$ $\left(\mathrm{CDCl}_{3}\right) \delta=1.09-1.19\left(\mathrm{~m}, \mathrm{C}-10\left(\mathrm{H}_{2}\right)\right.$, $\left.\mathrm{C}-11\left(\mathrm{H}_{2}\right), 4 \mathrm{H}\right) ; 1.69$ (s, SH, 1H); 1.86-2.20 (m, C-9(H $\left.\left.\mathrm{H}_{2}\right), 2 \mathrm{H}\right) ; 2.39$ (s, $\left.\underline{\mathrm{CH}}_{2} \mathrm{SH}, 2 \mathrm{H}\right) ; 2.85\left(\mathrm{t}, 2 \mathrm{C}-\mathrm{a}\left(\mathrm{H}_{2}\right), 4 \mathrm{H}\right)$; 3.44 (q, C-12(H), 1H); 3.85 (t, C$\left.8\left(\mathrm{H}_{2}\right), 2 \mathrm{H}\right) ; 4.15\left(\mathrm{t}, 2 \mathrm{C}-\mathrm{b}\left(\mathrm{H}_{2}\right), 4 \mathrm{H}\right)$; $5.18(\mathrm{~d}, \mathrm{C} .12 \mathrm{a}(\mathrm{H}), 1 \mathrm{H}) ; 7.26-7.71(\mathrm{~m}$, $\left.\mathrm{C}_{6} \mathrm{H}_{5}, 5 \mathrm{H}\right) ; 8.74$ (s, NH, 1H). 7a: MS: $\mathrm{m} / \mathrm{z}(\%)=\mathrm{M}^{+} 405$ (2.34), 160 (100).

\section{3-Methylsulfanylmethyl-12-mor-} pholino-5-phenyl-1,2,5,6,8,9,10,11, 12,12a-decahydropyrimido [4,5: 4,5]pyrimido[1,6-a]azepine-1,6dione (8a)

A mixture of $7 \mathrm{c}(10 \mathrm{mmol})$, methyl iodide (1.4 g, $10 \mathrm{mmol})$ and anhydrous potassium carbonate (1.9 $\mathrm{g}, 20 \mathrm{mmol})$ in dry acetone $(15 \mathrm{ml})$ was refluxed for about 6 hours and filtered while hot. The filtrate was distilled under vacuum; the residue was triturated with ethanol and the obtained crystals were filtered, dried and recrystallized from aqueous 
ethanol (Table IV). IR (KBr) 3236, 1620, 1595. ${ }^{1} \mathrm{H}-\mathrm{NMR}\left(\mathrm{CDCl}_{3}\right) \delta=$ $1.22-1.29\left(\mathrm{~m}, \mathrm{C}-10\left(\mathrm{H}_{2}\right), \mathrm{C}-11\left(\mathrm{H}_{2}\right)\right.$, $4 \mathrm{H}) ; \quad 1.55-1.82 \quad\left(\mathrm{~m}, \mathrm{C}-9\left(\mathrm{H}_{2}\right), 2 \mathrm{H}\right)$; $2.21\left(\mathrm{~s}, \mathrm{SCH}_{3}, 3 \mathrm{H}\right) ; 2.33$ (q, C-12(H), $1 \mathrm{H}) ; 2.51\left(\mathrm{~s}, \mathrm{CH}_{2} \mathrm{~S}, 2 \mathrm{H}\right): 2.87$ (t, 2C$\left.\mathrm{a}\left(\mathrm{H}_{2}\right), 4 \mathrm{H}\right) ; 3.26$ (t, C-8 $\left.\left(\mathrm{H}_{2}\right), 2 \mathrm{H}\right)$; $3.67\left(\mathrm{t}, 2 \mathrm{C}-\mathrm{b}\left(\mathrm{H}_{2}\right), 4 \mathrm{H}\right) ; 4.25(\mathrm{~d}, \mathrm{C}-12 \mathrm{a}$ $(\mathrm{H}), 1 \mathrm{H}) ; 7.12-7.63\left(\mathrm{~m}, \mathrm{C}_{6} \mathrm{H}_{5}, 5 \mathrm{H}\right)$; $9.35(\mathrm{~s}, \mathrm{NH}, 1 \mathrm{H})$.

5-Isopropyl- or phenyl-12-morpholino- or piperidino-1,6-dioxo$1,2,5,6,8,9,10,11,12,12 a-d e c a h y d r o-$ pyrimido $[4,5,: 4,5]$ pyrimido $[1,6-a]$ azepine-3-methyl thioacetic acid $(\mathbf{8 b}, \mathbf{c})$

A mixture of $7 \mathbf{a}, \mathbf{c}(10 \mathrm{mmol})$, chloroacetic acid $(0.94 \mathrm{ml}, 10 \mathrm{mmol})$ and potassium hydroxide $(0.72 \mathrm{~g}, 20$ mmol), in absolute ethanol $(20 \mathrm{ml})$ was refluxed for about 8 hours then filtered while hot. The filtrate was evaporated to dryness; the formed residue was dissolved in water then acidified with dilute hydrochloric acid. The separated crystals were filtered, washed with water and recrystallized from aqueous ethanol (Table IV). 8b: IR (KBr) 3421, 3275, 1652, 1598. 8c: IR (KBr) 3433, 3280, $1645,1612.8 b:{ }^{1} \mathrm{H}-\mathrm{NMR}\left(\mathrm{CDCl}_{3}\right): \delta=$ 1.16-1.34 (m, C-10 $\left(\mathrm{H}_{2}\right), \mathrm{C}-11\left(\mathrm{H}_{2}\right)$, $4 \mathrm{H}) ; 1.70\left(\mathrm{~d}, \mathrm{CH}\left(\mathrm{CH}_{3}\right)_{2}, 6 \mathrm{H}\right) ; 1.96-$ $2.21\left(\mathrm{~m}, \mathrm{C}-9\left(\mathrm{H}_{2}\right), 2 \mathrm{C}-\mathrm{b}\left(\mathrm{H}_{2}\right), \mathrm{C}-\mathrm{c}\left(\mathrm{H}_{2}\right)\right.$, $8 \mathrm{H}$ ); 2.73 (s, $\mathrm{CH}_{2} \mathrm{~S}, 2 \mathrm{H}$ ); 2.95 (t, 2C$\left.\mathrm{a}\left(\mathrm{H}_{2}\right), 4 \mathrm{H}\right) ; 3.53$ (septet, $\underline{\mathrm{CH}}\left(\mathrm{CH}_{3}\right)_{2}$, $1 \mathrm{H}) ; 4.35\left(\mathrm{t}, \mathrm{C}-8\left(\mathrm{H}_{2}\right), 2 \mathrm{H}\right) ; 4.56(\mathrm{~s}$, $\left.\mathrm{CH}_{2} \mathrm{COOH}, 2 \mathrm{H}\right) ; 5.07$ (d, C.12a(H), $1 \mathrm{H}) ; 5.53$ (q, C-12(H), 1H); 6.91 (s, $\mathrm{COOH}, 1 \mathrm{H}) ; 8.49(\mathrm{~s}, \mathrm{NH}, 1 \mathrm{H})$.
Ethyl 5-phenyl-12-piperidino-1,6dioxo-1,2,5,6,8,9,10,11,12,12a-decahydropyrimido $\left[4,5^{\prime}: 4,5\right]$ pyrimido [1,6-a]azepine-3-methyl thioacetate (9)

A mixture of compound $7 \mathbf{b}$ ( $4 \mathrm{~g}$, $10 \mathrm{mmol})$, ethyl chloroacetate $(0.9$ $\mathrm{ml}, \quad 10 \mathrm{mmol}$ ) and anhydrous potassium carbonate $(1.9 \mathrm{~g}, \quad 20$ $\mathrm{mmol})$, in dry acetone $(15 \mathrm{ml})$ was treated according to the method used for the preparation of compounds 8a (Table IV). IR (KBr) 3273, 1712, 1650, 1610. ${ }^{1} \mathrm{H}-\mathrm{NMR}\left(\mathrm{CDCl}_{3}\right) \quad \delta=$ 1.21-1.31 (m, C-10 $\left(\mathrm{H}_{2}\right), \mathrm{C}-11\left(\mathrm{H}_{2}\right)$, $4 \mathrm{H}) ; 1.73\left(\mathrm{t}, \quad \mathrm{COOCH}_{2} \mathrm{CH}_{3}, 3 \mathrm{H}\right)$; 1.96-2.21 (m, C-9 $\left(\mathrm{H}_{2}\right), 2 \mathrm{C}-\mathrm{b}\left(\mathrm{H}_{2}\right), \mathrm{C}-$ $\left.\mathrm{c}\left(\mathrm{H}_{2}\right), 8 \mathrm{H}\right) ; 2.55\left(\mathrm{~s}, \underline{\mathrm{CH}_{2} \mathrm{~S}}, 2 \mathrm{H}\right) ; 2.95$ $\left(\mathrm{t}, 2 \mathrm{C}-\mathrm{a}\left(\mathrm{H}_{2}\right), 4 \mathrm{H}\right) ; 3.23\left(\mathrm{~s}, \mathrm{CH}_{2} \mathrm{SCH}_{2}\right.$, $2 \mathrm{H}) ; 3.61\left(\mathrm{t}, \mathrm{C} 8\left(\mathrm{H}_{2}\right), 2 \mathrm{H}\right) ; 4.10(\mathrm{q}$, $\left.\mathrm{COOCH}_{2} \mathrm{CH}_{3}, \mathrm{C}-12(\mathrm{H}), 3 \mathrm{H}\right) ; 4.73(\mathrm{~d}$, $\mathrm{C}-12 \mathrm{a}(\mathrm{H}), 1 \mathrm{H}) ; 6.93-7.46\left(\mathrm{~m}, \mathrm{C}_{6} \mathrm{H}_{5}\right.$, $5 \mathrm{H}) ; 8.53(\mathrm{~s}, \mathrm{NH}, 1 \mathrm{H}) . \mathrm{MS}: \mathrm{m} / \mathrm{z}(\%)=$ $\mathrm{M}^{+} 525$ (3.97), 97 (100).

3-Acetyl or benzoyl sulfanylmethyl12-morpholino- or piperidino-5phenyl-1,2,5,6,8,9,10,11,12,12a-

decahydropyrimido $\left[4,5^{\prime}: 4,5\right]$ pyrimi do[1,6-a]azepine-1,6- dione (10a-c)

A mixture of $\mathbf{7 b}, \mathbf{c}(10 \mathrm{mmol})$, acetyl or benzoyl chloride $(11 \mathrm{mmol})$ and triethylamine $(0.5 \mathrm{ml})$ in dry benzene $(10 \mathrm{ml})$ was refluxed for about 4 hours. The solvent was removed under vacuum and the obtained residue was triturated with ether; the separated crystals were filtered and recrystallized from aqueous ethanol (Table IV). 10a: IR (KBr) $3235(\mathrm{NH})$, 1705, 1610 (2CO). 10c: IR (KBr) 3385 (NH), 1702 (CO), 
Table IV: Physical and analytical data of the prepared compounds 8a-c, 9, 10a-c, 14a-e and 15a-e.

\begin{tabular}{|c|c|c|c|c|c|c|c|c|}
\hline \multirow{2}{*}{ No } & \multirow{2}{*}{$\mathrm{R}$} & \multirow{2}{*}{$\mathrm{R}^{1}$} & \multirow{2}{*}{$\mathrm{R}^{2}$} & \multirow{2}{*}{$\begin{array}{l}\text { Yield \% } \\
\text { M.P }^{\circ}\end{array}$} & \multirow{2}{*}{$\begin{array}{l}\text { Mol. formula } \\
\text { (M.wt.) }\end{array}$} & \multicolumn{3}{|c|}{ Microanalytical data } \\
\hline & & & & & & & Cald. & Found \\
\hline $8 \mathbf{a}$ & $\mathrm{C}_{6} \mathrm{H}_{5}$ & & $\mathrm{CH}_{3}$ & $\begin{array}{c}65 \\
165-7\end{array}$ & $\begin{array}{c}\mathrm{C}_{23} \mathrm{H}_{29} \mathrm{~N}_{5} \mathrm{O}_{3} \mathrm{~S} \\
2 \mathrm{H}_{2} \mathrm{O} \\
491.61 \\
\end{array}$ & $\begin{array}{l}\mathrm{C} \\
\mathrm{H} \\
\mathrm{N} \\
\end{array}$ & $\begin{array}{c}56.19 \\
6.77 \\
14.25 \\
\end{array}$ & $\begin{array}{c}55.85 \\
6.52 \\
14.36 \\
\end{array}$ \\
\hline b & $\mathrm{CH}\left(\mathrm{CH}_{3}\right)_{2}$ & & $\mathrm{CH}_{2} \mathrm{COOH}$ & $\begin{array}{c}50 \\
205-7\end{array}$ & $\begin{array}{c}\mathrm{C}_{22} \mathrm{H}_{33} \mathrm{~N}_{5} \mathrm{O}_{4} \mathrm{~S} \\
463.60\end{array}$ & $\begin{array}{l}\mathrm{C} \\
\mathrm{H} \\
\mathrm{N}\end{array}$ & $\begin{array}{c}57.00 \\
7.17 \\
15.11\end{array}$ & $\begin{array}{c}57.31 \\
7.30 \\
15.64\end{array}$ \\
\hline c & $\mathrm{C}_{6} \mathrm{H}_{5}$ & & $\mathrm{CH}_{2} \mathrm{COOH}$ & $\begin{array}{c}60 \\
210-11\end{array}$ & $\begin{array}{c}\mathrm{C}_{24} \mathrm{H}_{29} \mathrm{~N}_{5} \mathrm{O}_{5} \mathrm{~S} \\
499.58\end{array}$ & $\begin{array}{l}\mathrm{C} \\
\mathrm{H} \\
\mathrm{N}\end{array}$ & $\begin{array}{c}57.70 \\
5.85 \\
14.02\end{array}$ & $\begin{array}{c}57.31 \\
5.38 \\
14.53\end{array}$ \\
\hline 9 & $\mathrm{C}_{6} \mathrm{H}_{5}$ & & $\mathrm{CH}_{2} \mathrm{COO} \mathrm{C}_{2} \mathrm{H}_{5}$ & $\begin{array}{c}70 \\
175-8\end{array}$ & $\begin{array}{c}\mathrm{C}_{27} \mathrm{H}_{35} \mathrm{~N}_{5} \mathrm{O}_{4} \mathrm{~S} \\
525.66\end{array}$ & $\begin{array}{l}\mathrm{C} \\
\mathrm{H} \\
\mathrm{N}\end{array}$ & $\begin{array}{c}61.69 \\
6.71 \\
13.32\end{array}$ & $\begin{array}{c}61.51 \\
6.91 \\
12.92\end{array}$ \\
\hline $10 \mathrm{a}$ & $\mathrm{C}_{6} \mathrm{H}_{5}$ & & $\mathrm{CH}_{3} \mathrm{CO}$ & $\begin{array}{c}60 \\
184-8\end{array}$ & $\begin{array}{c}\mathrm{C}_{25} \mathrm{H}_{31} \mathrm{~N}_{5} \mathrm{O}_{3} \mathrm{~S} \\
481.61\end{array}$ & $\begin{array}{l}\mathrm{C} \\
\mathrm{H} \\
\mathrm{N}\end{array}$ & $\begin{array}{c}62.35 \\
6.49 \\
14.54 \\
\end{array}$ & $\begin{array}{c}62.42 \\
6.25 \\
14.81 \\
\end{array}$ \\
\hline b & $\mathrm{C}_{6} \mathrm{H}_{5}$ & & $\mathrm{CH}_{3} \mathrm{CO}$ & $\begin{array}{c}65 \\
180-5\end{array}$ & $\begin{array}{c}\mathrm{C}_{24} \mathrm{H}_{29} \mathrm{~N}_{5} \mathrm{O}_{4} \mathrm{~S} . \\
1 / 2 \mathrm{H}_{2} \mathrm{O} \\
492.59\end{array}$ & $\begin{array}{l}\mathrm{C} \\
\mathrm{H} \\
\mathrm{N}\end{array}$ & $\begin{array}{c}58.52 \\
6.14 \\
14.22\end{array}$ & $\begin{array}{c}58.29 \\
5.81 \\
14.63\end{array}$ \\
\hline c & $\mathrm{C}_{6} \mathrm{H}_{5}$ & & $\mathrm{C}_{6} \mathrm{H}_{5} \mathrm{CO}$ & $\begin{array}{c}70 \\
195-7\end{array}$ & $\begin{array}{c}\mathrm{C}_{29} \mathrm{H}_{31} \mathrm{~N}_{5} \mathrm{O}_{4} \mathrm{~S} \\
545.66\end{array}$ & $\begin{array}{l}\mathrm{C} \\
\mathrm{H} \\
\mathrm{N}\end{array}$ & $\begin{array}{c}63.83 \\
5.73 \\
12.83 \\
\end{array}$ & $\begin{array}{c}63.44 \\
5.28 \\
12.53 \\
\end{array}$ \\
\hline $14 \mathbf{a}$ & $\mathrm{CH}\left(\mathrm{CH}_{3}\right)_{2}$ & & $\mathrm{CH}_{3}$ & $\begin{array}{c}45 \\
185-7\end{array}$ & $\begin{array}{c}\mathrm{C}_{21} \mathrm{H}_{31} \mathrm{~N}_{5} \mathrm{O}_{4} \\
417.50\end{array}$ & $\begin{array}{l}\mathrm{C} \\
\mathrm{H} \\
\mathrm{N} \\
\end{array}$ & $\begin{array}{c}60.41 \\
7.48 \\
16.77 \\
\end{array}$ & $\begin{array}{c}60.51 \\
7.63 \\
17.15 \\
\end{array}$ \\
\hline b & $\mathrm{C}_{6} \mathrm{H}_{5}$ & & $\mathrm{C}_{2} \mathrm{H}_{5}$ & $\begin{array}{c}65 \\
175-8\end{array}$ & $\begin{array}{c}\mathrm{C}_{25} \mathrm{H}_{31} \mathrm{~N}_{5} \mathrm{O}_{4} \\
465.55\end{array}$ & $\begin{array}{l}\mathrm{C} \\
\mathrm{H} \\
\mathrm{N}\end{array}$ & $\begin{array}{c}64.50 \\
6.71 \\
15.04\end{array}$ & $\begin{array}{c}64.16 \\
6.22 \\
15.60\end{array}$ \\
\hline c & $\mathrm{C}_{6} \mathrm{H}_{5}$ & & $\mathrm{CH}_{3}$ & $\begin{array}{c}75 \\
135-9\end{array}$ & $\begin{array}{c}\mathrm{C}_{23} \mathrm{H}_{27} \mathrm{~N}_{5} \mathrm{O}_{5} \\
453.49\end{array}$ & $\begin{array}{l}\mathrm{C} \\
\mathrm{H} \\
\mathrm{N} \\
\end{array}$ & $\begin{array}{c}60.92 \\
6.00 \\
15.44 \\
\end{array}$ & $\begin{array}{c}60.51 \\
5.85 \\
15.42 \\
\end{array}$ \\
\hline d & $\mathrm{C}_{6} \mathrm{H}_{5}$ & & $\mathrm{C}_{2} \mathrm{H}_{5}$ & $\begin{array}{c}80 \\
155-7\end{array}$ & $\begin{array}{c}\mathrm{C}_{24} \mathrm{H}_{29} \mathrm{~N}_{5} \mathrm{O}_{5} \\
467.52\end{array}$ & $\begin{array}{l}\mathrm{C} \\
\mathrm{H} \\
\mathrm{N} \\
\end{array}$ & $\begin{array}{c}61.66 \\
6.25 \\
14.98 \\
\end{array}$ & $\begin{array}{c}61.90 \\
6.78 \\
15.22 \\
\end{array}$ \\
\hline e & $\mathrm{C}_{6} \mathrm{H}_{5}$ & & $\mathrm{CH}\left(\mathrm{CH}_{3}\right)_{2}$ & $\begin{array}{c}85 \\
177-9\end{array}$ & $\begin{array}{c}\mathrm{C}_{25} \mathrm{H}_{31} \mathrm{~N}_{5} \mathrm{O}_{5} \\
1 / 2 \mathrm{H}_{2} \mathrm{O} \\
490.55\end{array}$ & $\begin{array}{l}\mathrm{C} \\
\mathrm{H} \\
\mathrm{N}\end{array}$ & $\begin{array}{c}61.21 \\
6.58 \\
14.28\end{array}$ & $\begin{array}{c}61.52 \\
6.31 \\
13.94\end{array}$ \\
\hline $15 \mathbf{a}$ & $\mathrm{C}_{6} \mathrm{H}_{5}$ & & & $\begin{array}{c}62 \\
203-5\end{array}$ & $\begin{array}{c}\mathrm{C}_{28} \mathrm{H}_{36} \mathrm{~N}_{6} \mathrm{O}_{3} \\
504.63\end{array}$ & $\begin{array}{l}\mathrm{C} \\
\mathrm{H} \\
\mathrm{N} \\
\end{array}$ & $\begin{array}{c}66.64 \\
7.19 \\
16.65 \\
\end{array}$ & $\begin{array}{c}66.22 \\
7.50 \\
16.35 \\
\end{array}$ \\
\hline
\end{tabular}


Table IV: (Cont.)

\begin{tabular}{|c|c|c|c|c|c|c|c|c|}
\hline \multirow{2}{*}{ No } & \multirow{2}{*}{$\mathrm{R}$} & \multirow{2}{*}{$\mathrm{R}^{1}$} & \multirow{2}{*}{$\mathrm{R}^{2}$} & \multirow{2}{*}{$\begin{array}{l}\text { Yield \% } \\
\text { M.P }^{\circ}\end{array}$} & \multirow{2}{*}{$\begin{array}{c}\text { Mol. formula } \\
\text { (M.wt.) }\end{array}$} & \multicolumn{3}{|c|}{ Microanalytical data } \\
\hline & & & & & & & Cald. & Found \\
\hline $15 b$ & $\mathrm{C}_{6} \mathrm{H}_{5}$ & & & $\begin{array}{c}70 \\
183-5\end{array}$ & $\begin{array}{c}\mathrm{C}_{30} \mathrm{H}_{34} \mathrm{~N}_{6} \mathrm{O}_{3} \\
526.63\end{array}$ & $\begin{array}{l}\mathrm{C} \\
\mathrm{H} \\
\mathrm{N}\end{array}$ & $\begin{array}{c}68.42 \\
6.51 \\
15.96\end{array}$ & $\begin{array}{c}68.14 \\
6.91 \\
16.38\end{array}$ \\
\hline c & $\mathrm{C}_{6} \mathrm{H}_{5}$ & & & $\begin{array}{c}75 \\
190-3\end{array}$ & $\begin{array}{c}\mathrm{C}_{27} \mathrm{H}_{34} \mathrm{~N}_{6} \mathrm{O}_{4} \\
506.60\end{array}$ & $\begin{array}{l}\mathrm{C} \\
\mathrm{H} \\
\mathrm{N}\end{array}$ & $\begin{array}{c}64.01 \\
6.76 \\
16.59\end{array}$ & $\begin{array}{c}64.30 \\
6.25 \\
16.37\end{array}$ \\
\hline d & $\mathrm{C}_{6} \mathrm{H}_{5}$ & & & $\begin{array}{c}55 \\
205-8\end{array}$ & $\begin{array}{c}\mathrm{C}_{28} \mathrm{H}_{30} \mathrm{~N}_{6} \mathrm{O}_{4} \\
514.58\end{array}$ & $\begin{array}{l}\mathrm{C} \\
\mathrm{H} \\
\mathrm{N}\end{array}$ & $\begin{array}{c}65.35 \\
5.88 \\
16.33\end{array}$ & $\begin{array}{c}65.10 \\
5.45 \\
16.17\end{array}$ \\
\hline $\mathbf{e}$ & $\mathrm{C}_{6} \mathrm{H}_{5}$ & & & $\begin{array}{c}65 \\
190-3\end{array}$ & $\begin{array}{c}\mathrm{C}_{26} \mathrm{H}_{32} \mathrm{~N}_{6} \mathrm{O}_{5} \\
508.58\end{array}$ & $\begin{array}{l}\mathrm{C} \\
\mathrm{H} \\
\mathrm{N}\end{array}$ & $\begin{array}{c}61.40 \\
6.34 \\
16.52\end{array}$ & $\begin{array}{c}61.83 \\
5.94 \\
16.30\end{array}$ \\
\hline
\end{tabular}

1615 (N-CO-N). 10a: ${ }^{1} \mathrm{H}-\mathrm{NMR}$ $\left(\mathrm{CDCl}_{3}\right) \delta=1.19-1.36\left(\mathrm{~m}, \mathrm{C}-10\left(\mathrm{H}_{2}\right)\right.$, $\left.\mathrm{C}-11\left(\mathrm{H}_{2}\right), \quad 4 \mathrm{H}\right) ; \quad 1.96-2.21 \quad(\mathrm{~m}, \mathrm{C}-$ $\left.9\left(\mathrm{H}_{2}\right), 2 \mathrm{C}-\mathrm{b}\left(\mathrm{H}_{2}\right), \mathrm{C}-\mathrm{c}\left(\mathrm{H}_{2}\right), 8 \mathrm{H}\right) ; 2.33$ $\left(\mathrm{s}, \mathrm{COCH}_{3}, 3 \mathrm{H}\right) ; 2.56\left(\mathrm{~s}, \mathrm{CH}_{2} \mathrm{~S}, 2 \mathrm{H}\right)$; $2.85\left(\mathrm{t}, 2 \mathrm{C}-\mathrm{a}\left(\mathrm{H}_{2}\right), 4 \mathrm{H}\right)$; 3.55 (q, C$12(\mathrm{H}), 1 \mathrm{H}) ; 4.03\left(\mathrm{t}, \mathrm{C}-8\left(\mathrm{H}_{2}\right), 2 \mathrm{H}\right)$; $4.73(\mathrm{~d}, \mathrm{C}-12 \mathrm{a}(\mathrm{H}), 1 \mathrm{H}) ; 7.43-8.26(\mathrm{~m}$, $\left.\mathrm{C}_{6} \mathrm{H}_{5}, 5 \mathrm{H}\right) ; 8.53$ (s, NH, 1H). 10c: ${ }^{1} \mathrm{H}-$ NMR (DMSO-d $)_{6} \delta=1.24-1.30(\mathrm{~m}$, C-10 $\left.\left(\mathrm{H}_{2}\right), \mathrm{C}-11\left(\mathrm{H}_{2}\right), 4 \mathrm{H}\right)$; 1.96-2.21 (m, C-9 $\left.\left(\mathrm{H}_{2}\right), 2 \mathrm{H}\right) ; 2.50\left(\mathrm{t}, 2 \mathrm{C}-\mathrm{a}\left(\mathrm{H}_{2}\right)\right.$, $4 \mathrm{H}) ; 2.81\left(\mathrm{~s}, \underline{\mathrm{CH}}_{2} \mathrm{~S}, 2 \mathrm{H}\right) ; 3.24(\mathrm{t}, \mathrm{C}-$ $\left.8\left(\mathrm{H}_{2}\right), 2 \mathrm{H}\right) ; 3.73\left(\mathrm{t}, 2 \mathrm{C}-\mathrm{b}\left(\mathrm{H}_{2}\right), 4 \mathrm{H}\right)$; 4.38 (q, C-12(H),1H); 4.76 (d, C$12 \mathrm{a}(\mathrm{H}), 1 \mathrm{H}) ; 7.13-7.66\left(\mathrm{~m}, 2 \mathrm{C}_{6} \mathrm{H}_{5}\right.$, $10 \mathrm{H}) ; 8.53$ (s, NH,1H). 10a: MS: m/z $(\%)=\mathrm{M}^{+} 481$ (6.22); 159 (100).

4-Chloro-N-[4-cyano-5-morpholino or piperidino-2-phenyl-1,2,4a,5,6,7, 8,9-octahydro-1-oxopyrimido[1,6-a] azepin-3-yl] butanamide (11a,b)

A mixture of $\mathbf{5 b}, \mathbf{c}(10 \mathrm{mmol})$, chlorobutyryl chloride $(2.1 \mathrm{ml}, 15$ mmol) and triethylamine $(2.5 \mathrm{ml})$ in dry benzene $(10 \mathrm{ml})$ was stirred for one hour. The reaction mixture was then refluxed for two hours then evaporated to dryness, under vacuum. The left residue was triturated with ether; the separated crystals were collected and recrystallized from aqueous ethanol (Table V). 11a: IR $\left(\mathrm{cm}^{-1}\right) 3277(\mathrm{NH}), 2205(\mathrm{CN}), 1729$, 1650 (2CO). 11b: IR $\left(\mathrm{cm}^{-1}\right) 3317$ (NH), $2215(\mathrm{CN}), 1725,1633$ (2CO). 11a: ${ }^{1} \mathrm{H}-\mathrm{NMR}$ (DMSO-d $\left.{ }_{6}\right) \delta=1.15$ $1.36\left(\mathrm{~m}, \mathrm{C}-6\left(\mathrm{H}_{2}\right), \mathrm{C}-7\left(\mathrm{H}_{2}\right), 4 \mathrm{H}\right) ; 1.75$ (quintet, $\mathrm{HNCOCH}_{2} \mathrm{CH}_{2-}, 2 \mathrm{H}$ ); 1.96$2.26\left(\mathrm{~m}, \mathrm{C}-8\left(\mathrm{H}_{2}\right), 2 \mathrm{C}-\mathrm{b}\left(\mathrm{H}_{2}\right), \mathrm{C}-\mathrm{c}\left(\mathrm{H}_{2}\right)\right.$, $8 \mathrm{H}) ; 2.59\left(\mathrm{t}, \mathrm{HNCOCH}_{2}-2 \mathrm{H}\right) ; 2.78$ $\left(\mathrm{t}, 2 \mathrm{C}-\mathrm{a}\left(\mathrm{H}_{2}\right), 4 \mathrm{H}\right) ; 3.29\left(\mathrm{t}, \mathrm{C}-9\left(\mathrm{H}_{2}\right)\right.$, $2 \mathrm{H}) ; 3.71\left(\mathrm{t},-\underline{\mathrm{CH}}_{2} \mathrm{Cl}, 2 \mathrm{H}\right) ; 4.34$ (q, C$5(\mathrm{H}), 1 \mathrm{H}) ; 4.73(\mathrm{~d}, \mathrm{C}-4 \mathrm{a}(\mathrm{H}), 1 \mathrm{H})$; 6.63-7.21(m, $\left.\mathrm{C}_{6} \mathrm{H}_{5}, 5 \mathrm{H}\right) ; 7.93$ (s, NH,1H). 11a MS: m/z(\%)= M -1469 (16.3); $\mathrm{M}^{+} 470$ (5.33), 133 (100). 
Table V: Physical and analytical data of the prepared compounds 11a,b, 12a,b and 13a,b.

\begin{tabular}{|c|c|c|c|c|c|c|}
\hline \multirow{2}{*}{ No } & \multirow{2}{*}{$\mathrm{R}$} & \multirow{2}{*}{$\begin{array}{c}\text { Yield \% } \\
\text { M.P }^{\circ}\end{array}$} & \multirow{2}{*}{$\begin{array}{l}\text { Mol. formula } \\
\text { (M.wt.) }\end{array}$} & \multicolumn{3}{|c|}{ Microanalytical data } \\
\hline & & & & & Calcd. & Found \\
\hline $11 \mathrm{a}$ & & $\begin{array}{c}60 \\
13-5\end{array}$ & $\begin{array}{c}\mathrm{C}_{25} \mathrm{H}_{32} \mathrm{ClN}_{5} \mathrm{O}_{2} \\
470.01\end{array}$ & $\begin{array}{l}\mathrm{C} \\
\mathrm{H} \\
\mathrm{N}\end{array}$ & $\begin{array}{c}63.89 \\
6.86 \\
14.90\end{array}$ & $\begin{array}{c}63.42 \\
6.51 \\
14.55\end{array}$ \\
\hline $\mathbf{b}$ & & $\begin{array}{c}73 \\
190-4\end{array}$ & $\begin{array}{c}\mathrm{C}_{24} \mathrm{H}_{30} \mathrm{ClN}_{5} \mathrm{O}_{3} \\
471.98\end{array}$ & $\begin{array}{l}\mathrm{C} \\
\mathrm{H} \\
\mathrm{N}\end{array}$ & $\begin{array}{c}61.07 \\
6.41 \\
14.84\end{array}$ & $\begin{array}{c}61.33 \\
6.56 \\
14.44\end{array}$ \\
\hline $12 a$ & & $\begin{array}{c}65 \\
125-8\end{array}$ & $\begin{array}{c}\mathrm{C}_{25} \mathrm{H}_{32} \mathrm{ClN}_{5} \mathrm{O}_{2} \\
470.01\end{array}$ & $\begin{array}{l}\mathrm{C} \\
\mathrm{H} \\
\mathrm{N}\end{array}$ & $\begin{array}{c}63.89 \\
6.86 \\
14.90\end{array}$ & $\begin{array}{c}63.72 \\
6.33 \\
14.61\end{array}$ \\
\hline $\mathbf{b}$ & & $\begin{array}{c}70 \\
150-3\end{array}$ & $\begin{array}{c}\mathrm{C}_{24} \mathrm{H}_{30} \mathrm{ClN}_{5} \mathrm{O}_{3} \\
471.98\end{array}$ & $\begin{array}{l}\mathrm{C} \\
\mathrm{H} \\
\mathrm{N}\end{array}$ & $\begin{array}{c}61.07 \\
6.41 \\
14.84\end{array}$ & $\begin{array}{c}61.35 \\
6.96 \\
14.53\end{array}$ \\
\hline $13 a$ & & $\begin{array}{c}65 \\
177-9\end{array}$ & $\begin{array}{c}\mathrm{C}_{25} \mathrm{H}_{31} \mathrm{~N}_{5} \mathrm{O}_{2} \\
433.55\end{array}$ & $\begin{array}{l}\mathrm{C} \\
\mathrm{H} \\
\mathrm{N}\end{array}$ & $\begin{array}{c}69.26 \\
7.21 \\
16.15\end{array}$ & $\begin{array}{c}69.31 \\
7.55 \\
16.37\end{array}$ \\
\hline $\mathbf{b}$ & & $\begin{array}{c}80 \\
180-5\end{array}$ & $\begin{array}{c}\mathrm{C}_{24} \mathrm{H}_{29} \mathrm{~N}_{5} \mathrm{O}_{3} \\
435.52\end{array}$ & $\begin{array}{l}\mathrm{C} \\
\mathrm{H} \\
\mathrm{N}\end{array}$ & $\begin{array}{c}66.19 \\
6.71 \\
16.08\end{array}$ & $\begin{array}{c}66.33 \\
7.14 \\
16.50\end{array}$ \\
\hline
\end{tabular}

3-Chloropropyl-12-morpholino- or piperidino-5-phenyl-1,2,5,6,8,9,10, 11,12,12a-decahydropyrimido[4, $\left.5^{\prime}: 4,5\right]$ pyrimido[1,6-a]azepine-1,6dione $(12 a, b)$

Compounds 11a,b were refluxed in alcoholic/ $\mathrm{HCl}$ solution $(15 \mathrm{ml})$ for three hours. The solvent was evaporated under vacuum; the left residue was triturated with ether and the separated crystals were collected and recrystallized from aqueous ethanol (Table V). 12a,: IR (KBr) 3244 (NH), 1725, 1637 (2CO). 12b: IR (KBr) $3205(\mathrm{NH}), 1710,1650$ (2CO). 12a: ${ }^{1} \mathrm{H}-\mathrm{NMR}$ (DMSO- $\mathrm{d}_{6}$ ) $\delta=$ 1.28-1.31 (m, C-10 $\left(\mathrm{H}_{2}\right), \mathrm{C}-11\left(\mathrm{H}_{2}\right)$, $4 \mathrm{H}) ; 1.42\left(\mathrm{t},-\mathrm{CH}_{2} \mathrm{CH}_{2} \mathrm{CH}_{2} \mathrm{Cl}, 2 \mathrm{H}\right)$;
1.55-1.66 (m, C-9 $\left(\mathrm{H}_{2}\right), 2 \mathrm{C}-\mathrm{b}\left(\mathrm{H}_{2}\right)$, C$\mathrm{c}\left(\mathrm{H}_{2}\right), \quad 8 \mathrm{H}$ ); $1.82 \quad$ (quintet,$\left.\mathrm{CH}_{2} \mathrm{CH}_{2} \mathrm{CH}_{2} \mathrm{Cl}, 2 \mathrm{H}\right) ; 2.83$ (t, 2C$\left.\mathrm{a}\left(\mathrm{H}_{2}\right), 4 \mathrm{H}\right) ; 2.98(\mathrm{q}, \mathrm{C}-12(\mathrm{H}), 1 \mathrm{H})$; $3.24\left(\mathrm{t}, \quad \mathrm{C}-8\left(\mathrm{H}_{2}\right), 2 \mathrm{H}\right) ; 3.62 \quad(\mathrm{t}$, $\left.\mathrm{CH}_{2} \mathrm{CH}_{2} \mathrm{CH}_{2} \mathrm{Cl}, 2 \mathrm{H}\right) ; 4.41$ (d, C$12 \mathrm{a}(\mathrm{H}), 1 \mathrm{H}) ; 7.25-7.71 \quad\left(\mathrm{~m}, \mathrm{C}_{6} \mathrm{H}_{5}\right.$, 5H); 9.50 (s, NH, 1H). 12b: ${ }^{1} \mathrm{H}-\mathrm{NMR}$ $\left(\right.$ DMSO- $\left._{6}\right) \quad \delta=1.15-1.36 \quad(\mathrm{~m}, \quad \mathrm{C}-$ $\left.10\left(\mathrm{H}_{2}\right), \mathrm{C}-11\left(\mathrm{H}_{2}\right), 4 \mathrm{H}\right) ; 1.41$ (t, $\mathrm{CH}_{2} \mathrm{CH}_{2} \mathrm{CH}_{2} \mathrm{Cl}, 2 \mathrm{H}$ ); 1.66 (quintet,$\left.\mathrm{CH}_{2} \mathrm{CH}_{2} \mathrm{CH}_{2} \mathrm{Cl}, 2 \mathrm{H}\right) ; 1.96-2.26$ (m, $\left.\mathrm{C}-9\left(\mathrm{H}_{2}\right), 2 \mathrm{H}\right) ; 2.73\left(\mathrm{t}, 2 \mathrm{C}-\mathrm{a}\left(\mathrm{H}_{2}\right), 4 \mathrm{H}\right)$; 3.29 (t, $\left.2 \mathrm{C}-\mathrm{b}\left(\mathrm{H}_{2}\right), \quad 4 \mathrm{H}\right) ; 3.55 \quad(\mathrm{t},-$ $\mathrm{CH}_{2} \mathrm{CH}_{2} \mathrm{CH}_{2} \mathrm{Cl}, 2 \mathrm{H}$ ); 3.70 (q, C$12(\mathrm{H}), 1 \mathrm{H}) ; 4.35\left(\mathrm{t}, \mathrm{C}-8\left(\mathrm{H}_{2}\right), 2 \mathrm{H}\right)$; 4.82 (brs, $\mathrm{C}-12 \mathrm{a}(\mathrm{H}), 1 \mathrm{H}) ; 6.63-7.21$ (m, $\left.\mathrm{C}_{6} \mathrm{H}_{5}, 5 \mathrm{H}\right) ; 7.93$ (s, NH, 1H). 
1-Morpholino- or piperidino-8phenyl-1,2,3,4,5,7,8,10,11,12,14, 14b-dodecahydropyrrolo[1, 2 : 1,2$]$ pyrimido $\left[4^{\prime}, 5^{\prime}: 4,5\right]$ pyrimido $[1,6-$ a]azepine-7,14-dione or

12-Morpholino- or piperidino-5phenyl-1,2,3,5,6,8,9,10,11,12,12a, 13-dodecahydro-pyrrolo $[2,1 ": 2,3]$ pyrimido $[4,5: 4,5]$ pyrimido $[1,6-a]$ azepine-6,13-dione, (13a,b)

A mixture of 12a,b $(10 \mathrm{mmol})$ and the appropriate amine $(11 \mathrm{mmol})$ in dry benzene $(15 \mathrm{ml})$ was refluxed for three hours. The reaction mixture was evaporated under vacuum and the obtained residue was triturated with ether. The separated crystals were collected and recrystallized from aqueous ethanol (Table V). 13a: IR (KBr) 1625, 1590 (2CO). 13b: IR (KBr) 1650, 1610 (2CO). 13a: ${ }^{1} \mathrm{H}-$ NMR $\left(\right.$ DMSO-d $\left.{ }_{6}\right) \delta=1.15-1.36(\mathrm{~m}$, C-10 $\left.\left(\mathrm{H}_{2}\right), \mathrm{C}-11\left(\mathrm{H}_{2}\right), 4 \mathrm{H}\right) ; 1.56$ (t, C$\left.1\left(\mathrm{H}_{2}\right), 2 \mathrm{H}\right) ; 1.75$ (quintet, $\mathrm{C}-2\left(\mathrm{H}_{2}\right)$, $2 \mathrm{H})$; $1.96-2.26$ (m, C-9 $\left(\mathrm{H}_{2}\right), \quad 2 \mathrm{C}-$ $\left.\mathrm{b}\left(\mathrm{H}_{2}\right), \mathrm{C}-\mathrm{c}\left(\mathrm{H}_{2}\right), 8 \mathrm{H}\right) ; 2.4\left(\mathrm{t}, 2 \mathrm{C}-\mathrm{a}\left(\mathrm{H}_{2}\right)\right.$, $4 \mathrm{H}) ; 2.89\left(\mathrm{t}, \mathrm{C}-3\left(\mathrm{H}_{2}\right), 2 \mathrm{H}\right), 3.73(\mathrm{t}, \mathrm{C}-$ $\left.8\left(\mathrm{H}_{2}\right), 2 \mathrm{H}\right) ; 4.35$ (q, C-12(H), $\left.1 \mathrm{H}\right)$; $4.73(\mathrm{~d}, \mathrm{C}-12 \mathrm{a}(\mathrm{H}), 1 \mathrm{H}) ; 6.63-7.21(\mathrm{~m}$, $\left.\mathrm{C}_{6} \mathrm{H}_{5}, 5 \mathrm{H}\right)$. 13a: $\mathrm{MS} \mathrm{m} / \mathrm{z}(\%): \mathrm{M}^{+} 433$ (14.03), 210 (100).13b: $\mathrm{MS} \mathrm{m} / \mathrm{z}(\%)=$ $\mathrm{M}^{+}-1434.1$ (0.02), 126.9 (100).

Alkyl 4-cyano-2-isopropyl- or phenyl-5-morpholino or piperidino1-oxo-1,2,4a,5,6,7,8,9-octahydropyrimido[1,6-a]azepin-3-ylcarbamoylformate (14a-e)

A mixture of $\mathbf{5 a - c}(10 \mathrm{mmol})$ and oxalyl chloride (1.9 g, $15 \mathrm{mmol})$ in dry benzene $(10 \mathrm{ml})$ was heated under reflux at a temperature $60-70^{\circ}$ for about two hours. The solvent and excess oxalyl chloride were evaporated under reduced pressure and the residue was washed with three portions of dry benzene $(5 \mathrm{ml})$ each. The appropriate alcohol $(20 \mathrm{ml})$ was added and the reaction mixture was heated under reflux for about two hours. The solvent was removed by distillation under reduced pressure and the obtained residue was crystallized from aqueous ethanol (Table IV). 14a: IR (KBr) 3221, 2211, 1738, 1652, 1598. 14b: IR (KBr) 3277, 2205, 1729, 1603. 14c: IR (KBr) 3217, 2215, 1735, 1613. 14d: IR (KBr) 3310, 2216, 1710, 1630. 14e: IR (KBr) 3250, 2218, 1734, 1620. 14a: ${ }^{1} \mathrm{H}-\mathrm{NMR}\left(\mathrm{CDCl}_{3}\right)$ $\delta=1.35-1.46\left(\mathrm{~m}, \mathrm{C}-6\left(\mathrm{H}_{2}\right), \mathrm{C}-7\left(\mathrm{H}_{2}\right)\right.$, $4 \mathrm{H}) ; 1.52\left(\mathrm{~d}, \mathrm{CH}\left(\underline{\mathrm{CH}}_{3}\right)_{2}, 6 \mathrm{H}\right) ; 1.82-$ $1.93\left(\mathrm{~m}, \mathrm{C}-8\left(\mathrm{H}_{2}\right), 2 \mathrm{C}-\mathrm{b}\left(\mathrm{H}_{2}\right), \mathrm{C}-\mathrm{c}(\mathrm{H} 2)\right.$, $8 \mathrm{H}) ; 2.53\left(\mathrm{t}, 2 \mathrm{C}-\mathrm{a}\left(\mathrm{H}_{2}\right), 4 \mathrm{H}\right) ; 3.24(\mathrm{t}$, $\left.\mathrm{C}-9\left(\mathrm{H}_{2}\right), 2 \mathrm{H}\right) ; 3.45(\mathrm{q}, \mathrm{C}-5(\mathrm{H}), 1 \mathrm{H})$; 3.67(s, $\left.\quad \mathrm{OCH}_{3}, \quad 3 \mathrm{H}\right) ; \quad 4.13$ (septet, $\left.\underline{\mathrm{CH}}\left(\mathrm{CH}_{3}\right)_{2}, 1 \mathrm{H}\right) ; 4.52(\mathrm{~d}, \mathrm{C}-4 \mathrm{a}(\mathrm{H})$, $1 \mathrm{H}) ; 8.72$ (s, NH, 1H). 14c: ${ }^{1} \mathrm{H}-\mathrm{NMR}$ $\left(\right.$ DMSO- $\left._{6}\right) \quad \delta=1.09-1.23 \quad(\mathrm{~m}, \mathrm{C}$ $\left.6\left(\mathrm{H}_{2}\right), \mathrm{C}-7\left(\mathrm{H}_{2}\right), 4 \mathrm{H}\right) ; 1.63-1.81(\mathrm{~m}, \mathrm{C}-$ $\left.8\left(\mathrm{H}_{2}\right), 2 \mathrm{H}\right) ; 2.45$ (t, 2C-a $\left.\left(\mathrm{H}_{2}\right), 4 \mathrm{H}\right)$; $2.88(\mathrm{q}, \mathrm{C}-5(\mathrm{H}), 1 \mathrm{H}) ; 3.52\left(\mathrm{~s}, \mathrm{OCH}_{3}\right.$, $3 \mathrm{H}) ; 3.69\left(\mathrm{t}, 2 \mathrm{C}-\mathrm{b}\left(\mathrm{H}_{2}\right), 4 \mathrm{H}\right) ; 4.19(\mathrm{t}$, C-9 $\left.\left(\mathrm{H}_{2}\right), 2 \mathrm{H}\right) ; 4.82$ (brs, C-4a(H), $1 \mathrm{H})$; 7.08-7.60 (m, $\left.\mathrm{C}_{6} \mathrm{H}_{5}, 5 \mathrm{H}\right) ; 9.38$ (s, NH, 1H). 14a: $\mathrm{MS} \mathrm{m} / \mathrm{z}(\%): \mathrm{M}^{+}$ 417 (4.21), 114 (100).

1N-(4-Cyano-5-morpholino- or piperidino-2-phenyl-1-oxo-1,2,4a,5,6,7, 8,9-octahydropyrimido $[1,6-a]$ azepin-3-yl)-2-morpholino- or piperidino-2-oxoacetamide (15a-e)

A mixture of $\mathbf{5 b}, \mathbf{c}(10 \mathrm{mmol})$ and oxalyl chloride (1.9 g, $15 \mathrm{mmol})$ in dry benzene $(5 \mathrm{ml})$ was refluxed at a 
temperature $60-70^{\circ}$ in a water bath for about two hours. The solvent and excess oxalyl chloride were evaporated under reduced pressure; the formed residue was dissolved in dry benzene $(15 \mathrm{ml})$ and the appropriate amine $(11 \mathrm{mmol})$ was added. The reaction mixture was refluxed for about three hours and evaporated to dryness; the residue was triturated with ethanol, filtered and recrystallized from aqueous ethanol (Table IV). 15a: 3315, 2220, 1656, 1598. 15b: IR (KBr) 3209, 2218, 1635, 1596. 15c: IR (KBr) 3280, 2217, 1649,1599. 15d: IR (KBr) 3285, 2221, 1655, 1610. 15e: IR (KBr) 3279, 2215, 1655,1598. 15a: ${ }^{1} \mathrm{H}-\mathrm{NMR}\left(\mathrm{CDCl}_{3}\right) \delta=1.21-1.39$ $\left(\mathrm{m}, \mathrm{C}-6\left(\mathrm{H}_{2}\right), \mathrm{C}-7\left(\mathrm{H}_{2}\right), 4 \mathrm{H}\right) ; 1.96-2.21$ (m, C-8 $\left(\mathrm{H}_{2}\right), 2 \mathrm{C}-\mathrm{b}\left(\mathrm{H}_{2}\right), 2 \mathrm{C}-\mathrm{b}^{\prime}\left(\mathrm{H}_{2}\right), \mathrm{C}-$ $\left.\mathrm{c}\left(\mathrm{H}_{2}\right), \quad \mathrm{C}-\mathrm{c}^{\prime}\left(\mathrm{H}_{2}\right), 14 \mathrm{H}\right) ; 2.95 \quad$ (t, 2C$\left.\mathrm{a}\left(\mathrm{H}_{2}\right), 4 \mathrm{H}\right) ; 3.26\left(\mathrm{t}, 2 \mathrm{C}-\mathrm{a}^{\prime}\left(\mathrm{H}_{2}\right), 4 \mathrm{H}\right)$; 3.73 (q, C-5(H), 1H); 4.35 (t, C9( $\left.\left.\mathrm{H}_{2}\right), 2 \mathrm{H}\right) ; 4.73(\mathrm{~d}, \mathrm{C}-4 \mathrm{a}(\mathrm{H}), 1 \mathrm{H})$; 6.93-7.46 (m, $\left.\mathrm{C}_{6} \mathrm{H}_{5}, 5 \mathrm{H}\right) ; 8.54$ (s, NH, 1H). 15b: ${ }^{1} \mathrm{H}-\mathrm{NMR}\left(\mathrm{CDCl}_{3}\right) \delta=1.21$ $1.41\left(\mathrm{~m}, \mathrm{C}-6\left(\mathrm{H}_{2}\right), \mathrm{C}-7\left(\mathrm{H}_{2}\right), 4 \mathrm{H}\right) ; 1.89$ (s, $\left.\mathrm{C}_{6} \mathrm{H}_{4}-\mathrm{CH}_{3}, 3 \mathrm{H}\right) ; 1.96-2.21$ (m, C8( $\left.\left(\mathrm{H}_{2}\right), 2 \mathrm{C}-\mathrm{b}\left(\mathrm{H}_{2}\right), \mathrm{C}-\mathrm{c}\left(\mathrm{H}_{2}\right), 8 \mathrm{H}\right) ; 2.95$ (t, $\left.2 \mathrm{C}-\mathrm{a}\left(\mathrm{H}_{2}\right), 4 \mathrm{H}\right) ; 3.71\left(\mathrm{t}, \mathrm{C}-9\left(\mathrm{H}_{2}\right)\right.$, $2 \mathrm{H}) ; 4.30$ (q, C-5(H), 1H); 4.83 (brs, $\mathrm{C}-4 \mathrm{a}(\mathrm{H}), 1 \mathrm{H})$; 7.03-7.66 (m, $\mathrm{C}_{6} \mathrm{H}_{5}$, $\left.\mathrm{C}_{6} \mathrm{H}_{4}, 9 \mathrm{H}\right) ; 9.33$ (s, NH,1H). 15e: ${ }^{1} \mathrm{H}-$ NMR (DMSO- $\left.{ }_{6}\right): \delta=1.29-1.59(\mathrm{~m}$, C-6 $\left.\left(\mathrm{H}_{2}\right), \mathrm{C}-7\left(\mathrm{H}_{2}\right), 4 \mathrm{H}\right) ; 1.66-1.80(\mathrm{~m}$, $\left.\mathrm{C}-8\left(\mathrm{H}_{2}\right), 2 \mathrm{H}\right) ; 2.50\left(\mathrm{t}, 2 \mathrm{C}-\mathrm{a}\left(\mathrm{H}_{2}\right), 4 \mathrm{H}\right)$; 3.05 (t, 2C-a' $\left.\left(\mathrm{H}_{2}\right), 4 \mathrm{H}\right) ; 3.45$ (t, C$\left.9\left(\mathrm{H}_{2}\right), 2 \mathrm{H}\right) ; 3.73\left(\mathrm{t}, 2 \mathrm{C}-\mathrm{b}\left(\mathrm{H}_{2}\right), 2 \mathrm{C}-\right.$ b' $\left.\left(\mathrm{H}_{2}\right), 8 \mathrm{H}\right) ; 4.35(\mathrm{q}, \mathrm{C}-5(\mathrm{H}), 1 \mathrm{H})$; $4.80(\mathrm{~d}, \mathrm{C}-4 \mathrm{a}(\mathrm{H}), 1 \mathrm{H}) ; 7.23-7.96(\mathrm{~m}$, $\left.\mathrm{C}_{6} \mathrm{H}_{5}, 5 \mathrm{H}\right) ; 8.83$ (s, NH, 1H). 15a:
MS m/z (\%): $\mathrm{M}^{+} 504$ (2.11), 149 (100).

Alkyl 12-morpholino-5-phenyl-1,6dioxo-1,2,5,6,8,9,10,11,12,12a-decahydropyrimido $[4,5: 4,5]$ pyrimido [1,6-a]azepine-3-carboxylate $(16 a, b)$

Compounds 14c,d $(10 \mathrm{mmol})$ were refluxed with sodium ethoxide (sodium metal $0.2 \mathrm{~g}$ in ethanol $15 \mathrm{ml}$ ) for 10 hours then left to cool at room temperature.The separated crystals were filtered, washed with water and recrystallized from aqueous ethanol (Table VI). 16a: IR (KBr) 3245, 1734, 1625. 16b: IR (KBr) 3277, 1729, 1603. 16a: ${ }^{1} \mathrm{H}-\mathrm{NMR}\left(\mathrm{CDCl}_{3}\right)$ $\delta=1.12-1.32\left(\mathrm{~m}, \mathrm{C}-10\left(\mathrm{H}_{2}\right), \mathrm{C}-11\left(\mathrm{H}_{2}\right)\right.$, $4 \mathrm{H})$; $1.53-1.86\left(\mathrm{~m}, \mathrm{C}-9\left(\mathrm{H}_{2}\right), 2 \mathrm{H}\right)$; 2.34 (q, C-12(H), 1H); 2.87 (t, 2C$\left.\mathrm{a}\left(\mathrm{H}_{2}\right), 4 \mathrm{H}\right) ; 3.16\left(\mathrm{t}, \mathrm{C}-8\left(\mathrm{H}_{2}\right), 2 \mathrm{H}\right)$; $3.67\left(\mathrm{~s}, \mathrm{OCH}_{3}, 3 \mathrm{H}\right) ; 4.07$ (t, $2 \mathrm{C}-$ $\left.\mathrm{b}\left(\mathrm{H}_{2}\right), 4 \mathrm{H}\right) ; 4.43(\mathrm{~d}, \mathrm{C}-12 \mathrm{a}(\mathrm{H}), 1 \mathrm{H})$ : 7.13-7.64 (m, $\left.\mathrm{C}_{6} \mathrm{H}_{5}, 5 \mathrm{H}\right) ; 9.22$ (s, $\mathrm{NH}$, 1H). 16b: ${ }^{1} \mathrm{H}-\mathrm{NMR}$ (DMSO- $\left.\mathrm{d}_{6}\right) \delta=$ 1.01-1.23 (m, C-10 $\left(\mathrm{H}_{2}\right), \mathrm{C}-11\left(\mathrm{H}_{2}\right)$, $4 \mathrm{H}) ; 1.47\left(\mathrm{t}, \mathrm{OCH}_{2} \mathrm{CH}_{3}, 3 \mathrm{H}\right) ; 1.83-$ $2.01\left(\mathrm{~m}, \mathrm{C}-9\left(\mathrm{H}_{2}\right), 2 \mathrm{H}\right) ; 2.47$ (t, $2 \mathrm{C}-$ $\left.\mathrm{a}\left(\mathrm{H}_{2}\right), 4 \mathrm{H}\right) ; 3.13\left(\mathrm{t}, \mathrm{C}-8\left(\mathrm{H}_{2}\right), 2 \mathrm{H}\right)$; $3.53\left(\mathrm{t}, 2 \mathrm{C}-\mathrm{b}\left(\mathrm{H}_{2}\right), 4 \mathrm{H}\right) ; 4.31 \quad(\mathrm{q}$, $\left.\mathrm{OCH}_{2} \mathrm{CH}_{3}, \mathrm{C}-12(\mathrm{H}), 3 \mathrm{H}\right) ; 4.82(\mathrm{~d}, \mathrm{C}-$ $12 \mathrm{a}(\mathrm{H}), 1 \mathrm{H}) ; 7.16-7.60 \quad\left(\mathrm{~m}, \mathrm{C}_{6} \mathrm{H}_{5}\right.$, $5 \mathrm{H}) ; 9.18$ (s, NH, 1H). 16a: $\mathrm{MS} \mathrm{m} / \mathrm{z}$ (\%): $\mathrm{M}^{+} 453$ (5.21), 161 (100).

12-Morpholino- or piperidino-5phenyl-1,6-dioxo-1,2,5,6,8,9,10,11, 12,12a.decahydro-pyrimido[ $4,5: 4$, 5]pyrimido[1,6-a]azepine-3-substituted carboxamide $(17 \mathbf{a}, \mathbf{b})$

Compounds 15c,e $(10 \mathrm{mmol})$ were refluxed in alcoholic/ $\mathrm{HCl}$ solution (15 $\mathrm{ml}$ ) for about three hours then left to 
Table VI: Physical and analytical data of the prepared compounds 16a,b and $17 \mathbf{a}, \mathbf{b}$.

\begin{tabular}{|c|c|c|c|c|c|c|c|}
\hline \multirow{2}{*}{ No } & \multirow{2}{*}{$\mathrm{R}^{1}$} & \multirow{2}{*}{$\mathrm{R}^{2}$} & \multirow{2}{*}{$\begin{array}{l}\text { Yield \% } \\
\text { M.Po }\end{array}$} & \multirow{2}{*}{$\begin{array}{c}\text { Mol. } \\
\text { formula } \\
\text { (M.wt.) }\end{array}$} & \multicolumn{3}{|c|}{ Microanalytical data } \\
\hline & & & & & & Calcd. & Found. \\
\hline $16 \mathbf{a}$ & & $\mathrm{CH}_{3}$ & $\begin{array}{c}70 \\
202-5\end{array}$ & $\begin{array}{c}\mathrm{C}_{23} \mathrm{H}_{27} \mathrm{~N}_{5} \mathrm{O}_{5} \\
453.49\end{array}$ & $\begin{array}{l}\mathrm{C} \\
\mathrm{H} \\
\mathrm{N}\end{array}$ & $\begin{array}{c}60.92 \\
6.00 \\
15.44\end{array}$ & $\begin{array}{c}60.53 \\
6.20 \\
14.95\end{array}$ \\
\hline b & & $\mathrm{C}_{2} \mathrm{H}_{5}$ & $\begin{array}{c}65 \\
195-7\end{array}$ & $\begin{array}{c}\mathrm{C}_{24} \mathrm{H}_{29} \mathrm{~N}_{5} \mathrm{O}_{5} \\
467.52\end{array}$ & $\begin{array}{l}\mathrm{C} \\
\mathrm{H} \\
\mathrm{N}\end{array}$ & $\begin{array}{c}61.66 \\
6.25 \\
14.98 \\
\end{array}$ & $\begin{array}{c}61.44 \\
6.13 \\
15.27\end{array}$ \\
\hline $17 a$ & & & $\begin{array}{c}65 \\
165-8\end{array}$ & $\begin{array}{c}\mathrm{C}_{27} \mathrm{H}_{34} \mathrm{~N}_{6} \mathrm{O}_{4} \\
506.60\end{array}$ & $\begin{array}{l}\mathrm{C} \\
\mathrm{H} \\
\mathrm{N}\end{array}$ & $\begin{array}{c}64.01 \\
6.76 \\
16.59 \\
\end{array}$ & $\begin{array}{c}64.41 \\
6.65 \\
16.81 \\
\end{array}$ \\
\hline b & & & $\begin{array}{c}80 \\
180-3\end{array}$ & $\begin{array}{c}\mathrm{C}_{26} \mathrm{H}_{32} \mathrm{~N}_{6} \mathrm{O}_{5} \\
508.57\end{array}$ & $\begin{array}{l}\mathrm{C} \\
\mathrm{H} \\
\mathrm{N} \\
\end{array}$ & $\begin{array}{c}61.40 \\
6.34 \\
16.52 \\
\end{array}$ & $\begin{array}{c}61.22 \\
6.49 \\
16.73 \\
\end{array}$ \\
\hline
\end{tabular}

cool. The separated crystals were filtered and recrystallized from aqueous ethanol (Table VI). 17a: IR (KBr) $3245(\mathrm{NH}), 1637,1598(2 \mathrm{CO})$. 17b: IR (KBr) 3233 (NH), 1621, 1596 (2CO). 17a: ${ }^{1} \mathrm{H}-\mathrm{NMR}$ (DMSO$\left.\mathrm{d}_{6}\right) \delta=1.01-1.23\left(\mathrm{~m}, \mathrm{C}-10\left(\mathrm{H}_{2}\right), \mathrm{C}-\right.$ $\left.11\left(\mathrm{H}_{2}\right), 4 \mathrm{H}\right) ; 1.63-1.81\left(\mathrm{~m}, \mathrm{C}-9\left(\mathrm{H}_{2}\right)\right.$, 2C-b'(H2), C-c(H2), 8H); 2.45 (t, 2C$\left.\mathrm{a}\left(\mathrm{H}_{2}\right), 4 \mathrm{H}\right) ; 3.18\left(\mathrm{t}, 2 \mathrm{C}-\mathrm{a}^{\prime}\left(\mathrm{H}_{2}\right), 4 \mathrm{H}\right)$; $3.72\left(\mathrm{t}, \mathrm{C}-8\left(\mathrm{H}_{2}\right), 2 \mathrm{H}\right) ; 4.20$ (quintet, $\left.2 \mathrm{C}-\mathrm{b}\left(\mathrm{H}_{2}\right), 4 \mathrm{H}\right) ; 4.76$ (q, C-12(H), $1 \mathrm{H}) ; 5.27(\mathrm{~d}, \mathrm{C}-12 \mathrm{a}(\mathrm{H}), 1 \mathrm{H}) ; 7.16-$ $7.64\left(\mathrm{~m}, \mathrm{C}_{6} \mathrm{H}_{5}, 5 \mathrm{H}\right) ; 9.25$ (s, NH, 1H).

\section{Anti-inflammatory screening}

The anti-inflammatory activity of 18 selected newly synthesized compounds $4 c, 5 c, 6 c, 7 c, 8 c, 10 b, c$, 11b, 12b, 13b, 14c,d,e, 15c,d,e, 16b, 17a was evaluated using the method of "rat paw carrageenan oedema" as described by Winter $e t a l .{ }^{4}$

\section{Procedure}

Adult albino rats of both sexes weighing between 120-150 g were used. Rats were uniformly hydrated by giving $3 \mathrm{ml}$ water/rat through gastric inculation to reduce variability to oedema response. Animals were divided into 20 groups each of six animals. The control group was given saline solution containing few drops of Tween 80. Diclofenac sodium (10 $\mathrm{mg} / \mathrm{Kg}$ ) and drugs under examination $(10 \mathrm{mg} / \mathrm{Kg})$ were suspended in distilled water by the aid of few drops of Tween 80 (to improve wettability of the particles) and were given intraperitoneally one hour before induction of inflammation. Induction of inflammation was performed by s.c injection of 501 of $1 \%$ carargeenansodium gel into the subplantar region. The volume of the inflamed paw was measured just before and three hours after induction of inflammation (Table VII). 
Table VII: Anti-inflammatory effects of the tested compounds using the rat paw carrageenan oedema technique.

\begin{tabular}{|c|c|c|c|c|c|c|c|c|c|}
\hline \multirow{2}{*}{ Compound } & \multirow{2}{*}{$\begin{array}{l}\text { Dose } \\
\mathrm{mg} / \mathrm{kg}\end{array}$} & \multicolumn{6}{|c|}{ Decrease in oedema weight $(\mathrm{g})$} & \multirow{2}{*}{$\begin{array}{c}\text { Mean oedema } \\
\text { weight } \pm \text { SE }\end{array}$} & \multirow{2}{*}{$\begin{array}{c}\% \\
\text { Inhibition }\end{array}$} \\
\hline & & 1 & 2 & 3 & 4 & 5 & 6 & & \\
\hline Control & 0 & 0.8 & 0.9 & 0.5 & 0.3 & 0.9 & 0.9 & $0.7 \pm 0.23$ & \\
\hline Diclofenac-Na & 10 & 0.2 & 0.1 & 0.2 & 0.2 & 0.1 & 0.2 & $0.16 \pm 0.05$ & 77.1 \\
\hline $4 c$ & 10 & 0 & 0 & 0.1 & 0 & 0.2 & 0.2 & $0.08 \pm 0.09$ & 88.5 \\
\hline $5 c$ & 10 & 0.2 & 0 & 0.4 & 0.2 & 0.3 & 0.2 & $0.21 \pm 0.13$ & 70.0 \\
\hline $6 c$ & 10 & 0 & 0.2 & 0.5 & 0.4 & 0.4 & 0.2 & $0.28 \pm 0.18$ & 60.0 \\
\hline $7 \mathrm{c}$ & 10 & 0.4 & 0.3 & 0.0 & 0.3 & 0.4 & 0.4 & $0.3 \pm 0.15$ & 57.1 \\
\hline $8 \mathrm{c}$ & 10 & 0.0 & 0.3 & 0.1 & 0.1 & 0.2 & 0.1 & $0.13 \pm 0.10$ & 81.4 \\
\hline $10 \mathrm{~b}$ & 10 & 0.2 & 0.1 & 0.2 & 0.2 & 0.3 & 0.1 & $0.18 \pm 0.08$ & 74.2 \\
\hline $10 \mathrm{c}$ & 10 & 0.2 & 0.2 & 0.0 & 0.3 & 0.4 & 0.1 & $0.2 \pm 0.13$ & 71.4 \\
\hline 11b & 10 & 0.4 & 0.2 & 0.4 & 0.3 & 0.1 & 0.2 & $0.26 \pm 0.12$ & 63.3 \\
\hline $12 \mathrm{~b}$ & 10 & 0.4 & 0.3 & 0.3 & 0.2 & 0.4 & 0.4 & $0.33 \pm 0.08$ & 53.5 \\
\hline $13 \mathrm{~b}$ & 10 & 0.7 & 0.3 & 0.5 & 0.4 & 0.3 & 0.3 & $0.41 \pm 0.16$ & 41.4 \\
\hline $14 c$ & 10 & 0.1 & 0.1 & 0.1 & 0.1 & 0.2 & 0.1 & $0.11 \pm 0.04$ & 84.2 \\
\hline 14d & 10 & 0.1 & 0 & 0.2 & 0.2 & 0.3 & 0 & $0.13 \pm 0.12$ & 81.0 \\
\hline $14 \mathrm{e}$ & 10 & 0.5 & 0.3 & 0.1 & 0.4 & 0.1 & 0.3 & $0.28 \pm 0.14$ & 60.0 \\
\hline $15 \mathrm{c}$ & 10 & 0.3 & 0.2 & 0.3 & 0.3 & 0.2 & 0.2 & $0.25 \pm 0.05$ & 64.2 \\
\hline $15 d$ & 10 & 0.8 & 0.3 & 0.4 & 0.1 & 0.3 & 0.0 & $0.31 \pm 0.27$ & 55.7 \\
\hline $15 \mathrm{e}$ & 10 & 0.6 & 0.4 & 0.2 & 0.5 & 0.2 & 0.4 & $0.38 \pm 0.16$ & 46.4 \\
\hline $16 \mathrm{~b}$ & 10 & 0.5 & 0.2 & 0.6 & 0.6 & 0.3 & 0.0 & $0.36 \pm 0.24$ & 49.2 \\
\hline $17 \mathbf{a}$ & 10 & 0.4 & 0.2 & 0.4 & 0.3 & 0.1 & 0.2 & $0.26 \pm 0.12$ & 55.7 \\
\hline
\end{tabular}

The percentage inhibition of inflammation was calculated according to the following equation: $\%$ Inhibition $=\left(1-\mathrm{w}_{\mathrm{t}} / \mathrm{w}_{\mathrm{c}}\right) \times 100$.

\section{RESULTS AND DISCUSSION}

\section{Synthesis}

The starting compound 2dicyanomethylidenoperhydroazepine $\mathbf{1}^{3 \& 5}$ was treated with sulphuryl chloride according to a previously reported method ${ }^{2 \& 6}$ yielding the 3chloro-derivative (2).

In previous work, the preparation of the o-iminonitriles from the dicyanomethylidene derivative of azepine was described. ${ }^{1,3,7 \& 8}$ Thereby, in the present work refluxing compound $\mathbf{2}$ with isopropyl or phenyl isocyanate in methylene chloride using triethylamine as a catalyst provided compounds $\mathbf{3 a}, \mathbf{b}^{2}$ (Scheme 1).

Good yields of the 3-imino-2isopropyl or phenyl-5-morpholino or piperidino-1,2,3,5,6,7,8,9-octahydro1-oxopyrimido[1,6-a]azepine-4-carbonitrile 4a-c were obtained when the chloro derivatives $\mathbf{3 a}, \mathbf{b}$ were reacted with either morpholine or piperidine in absolute ethanol. 
<smiles>N#CC(C#N)=C(Cl)/C(C#N)=C1\NCCCCC1=C(C#N)C#N</smiles>

1

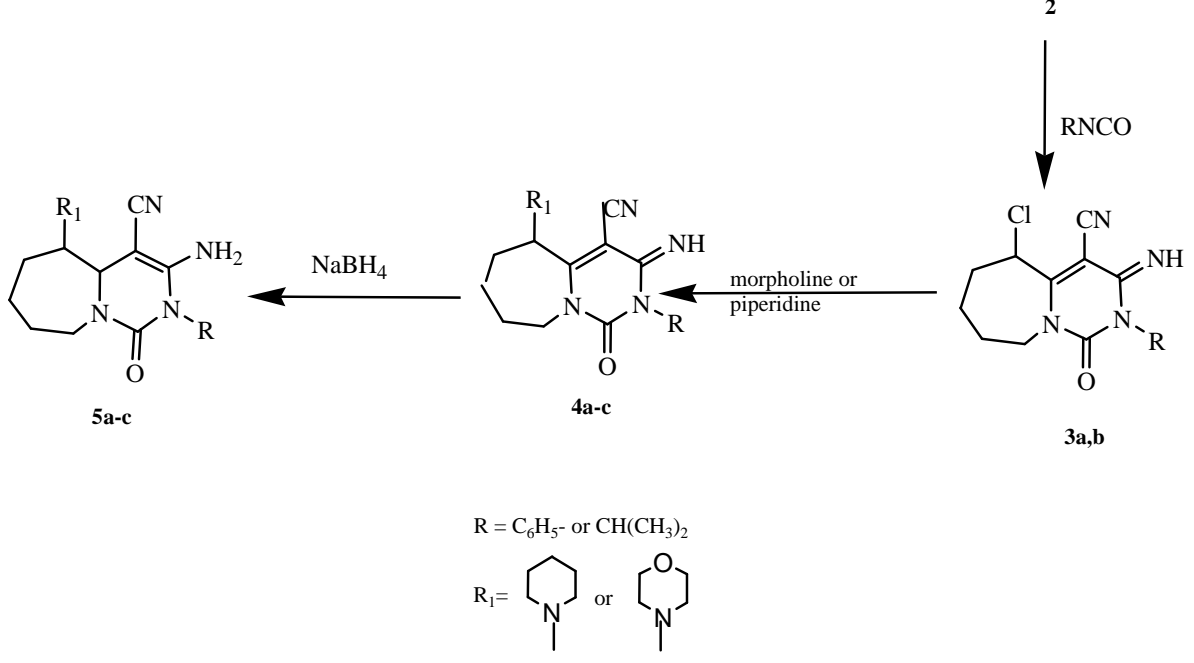

The corresponding 3-amino analogues 5a-c were obtained through 1,4 -addition reaction ${ }^{1,9 \& 10}$ of one molecule of hydrogen, achieved by the action of sodium borohydride on the 3-imino derivatives $\mathbf{4 a - c}$ in absolute ethanol (Scheme 1).

Preparation of 3-chloromethyl-5isopropyl or phenyl-12-morpholino or piperidino $1,2,5,6,8,9,10,11,12,12 \mathrm{a}-$ decahydropyrimido[4',5',4,5]pyrimid o[1,6-a]azepine-1,6-diones 6a-c was achieved $^{1,11-14}$ by refluxing the enaminonitrile derivatives 5a-c with chloroacetyl chloride, in dry benzene, using triethylamine as a base catalyst. The data drawn from IR spectra of $\mathbf{6}$ supported the proposed tricyclic structure due to the disappearance of the nitrile absorption band. The suggested mechanism was previously reported. $^{1 \& 15}$
Decomposition of the methyl isothiourea salts, obtained from the interaction of thiourea with the 3chloromethyl derivatives 6a-c yielded the corresponding 3-thiomethyl derivatives 7a-c.

Preparation of the corresponding methyl thioether 8a was done through the reaction of the thiol, compound $7 \mathbf{c}$ with methyl iodide and anhydrous potassium carbonate, in dry acetone.

The methylthioacetic acid derivatives $\mathbf{8 b}, \mathbf{c}$ were prepared through reacting the mercapto derivatives 7a,c with chloroacetic acid and potassium hydroxide, in absolute ethanol. Moreover, refluxing 7b with ethylchloroacetate and anhydrous potassium carbonate in dry acetone afforded the methylthioacetate derivative (9). 
A simple method was done to prepare the thioesters 10a-c through refluxing the thiol, compounds $\mathbf{7 b}, \mathbf{c}$ with acetyl or benzoyl chloride, in dry benzene and using triethylamine as a catalyst (Scheme 2).

Applying our previous method of chloroacylation, the enaminonitriles $\mathbf{5 b}, \mathbf{c}$ were reacted with chlorobutyryl chloride in dry benzene, using triethylamine as a catalyst, affording unexpectedly the uncyclized 3-(4chlorobutanamide) derivatives 11a,b. Evidence that intramolecular cyclization didn't occur was drawn from the fact that IR scanning of the obtained products showed a strong and sharp nitrile stetching band at $2205 \mathrm{~cm}^{-1}$.

Thereby, in a successful trial to obtain the tricyclic derivatives, compounds 11a,b were refluxed with ethanolic hydrochloric acid solution for three hours yielding 3chloropropyl-12-morpholino or piperidino-5-phenyl-1,2,5,6,8,9,10,11, 12,12a-decahydropyrimido[4,5:4,5] pyrimido[1,6-a]azepine-1,6-diones,

12a,b. The proposed structure of $\mathbf{1 2}$ was inferred from their IR spectra, which revealed the absence of the cyano group.

Furthermore, refluxing the tricyclic compounds 12a,b with different alicyclic secondary amines as well as primary aromatic amines afforded $^{16}$ a single crystalline compound identified as the tetracyclic ring system pyrrolopyrimidopyrimido-azepines 13a,b as confirmed by physical properties, thin layer chromatography, elemental, as well as spectral analyses (Scheme 3). The lack of a stretching band around 3200 $\mathrm{cm}^{-1}$ gave us a support for the intramolecular cyclization onto the imidic nitrogen leading to formation of the tetracycles. Additionally, the ${ }^{1} \mathrm{H}-\mathrm{NMR}$ spectrum of 13a revealed a triplet at $2.89 \mathrm{ppm}$, quintet at 1.75 $\mathrm{ppm}$ and a triplet at $1.56 \mathrm{ppm}$ related to the methylene protons of the pyrrole ring as compared to a triplet at $3.62 \mathrm{ppm}$, quintet at $1.82 \mathrm{ppm}$ and a triplet at $1.42 \mathrm{ppm}$ related to the corresponding methylene protons of the 3-chloropropyl moiety observed in the ${ }^{1}$ H-NMR scan of 12a. Moreover, mass spectrum of 13b showed the molecular ion peak at 434.1 constituting the molecular weight of the compound -1 .

In the above reaction two possible structural isomers (linear and angular) could be obtained.

As previously discussed and similar to other acid chlorides, oxalyl chloride was reacted with the enaminonitriles 5a-c in dry benzene aiming for an additional fused pyrimidine ring as previously reported. ${ }^{17}$ However, the high instability and rapid hydrolysis of the formed chlorocarbonyl derivatives made their isolation difficult. Accordingly, the crude products, obtained from the reaction of $\mathbf{5 a - c}$ with oxalyl chloride, were smoothly reacted with different alcohols yielding crystalline compounds identified, unexpectedly, as the uncyclized carbamoylformate derivatives 14a-e as drawn from the IR spectra of these compounds which demonstrated a strong nitrile absorption band. 


\section{Scheme2}
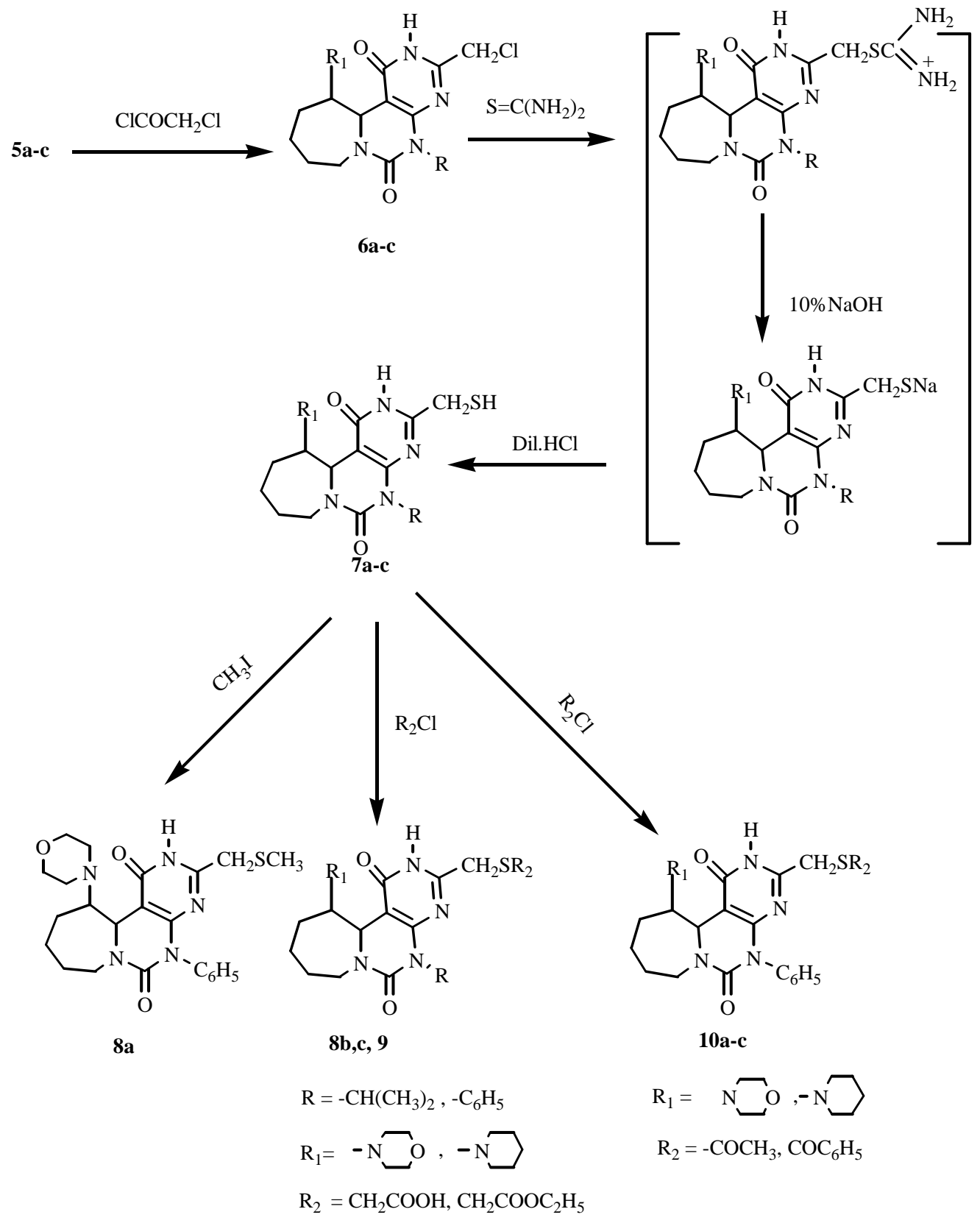


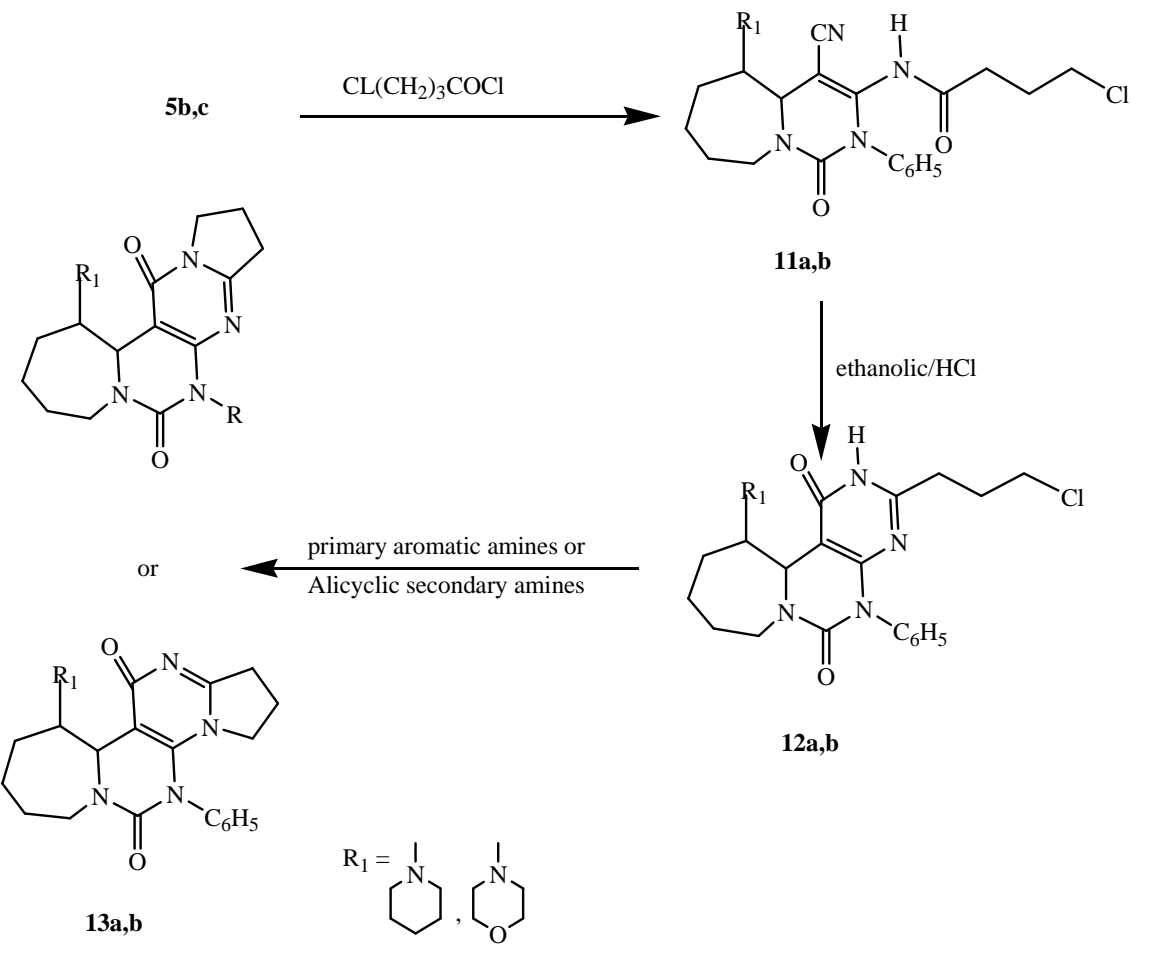

Furthermore, amide formation was carried out via the reaction of the acid chloride intermediates with excess of the appropriate amine using dry benzene, as a solvent, to produce the corresponding N-substituted-2oxoacetamide derivatives 15a-e. The IR spectra of these compounds revealed the presence of the characteristic nitrile stretching band.

The carbamoylformate derivatives $\mathbf{1 4 c , d}$ were cyclized via refluxing with sodium ethoxide to produce the desired tricycles 16a,b. Moreover, preparation of N-substituted carboxamides 17a,b was achieved when the corresponding $\mathrm{N}$-substituted-2-oxoacetamide derivatives $\mathbf{1 5 c , d}$ were refluxed with alcoholic solution of hydrochloric acid (Scheme 4). IR spectra of compounds 16 and $\mathbf{1 7}$ were complying with their structures.

\section{Pharmacological properties}

Application of one way ANOVA statistical test on the above tabulated data as well as the graphical representation (Fig. 1) of these data indicate that all the 18 newly synthesized compounds have significant anti-inflammatory effect at $\mathrm{P}<0.05$. On the other hand, significant activity at $\mathrm{P}<0.01$ was assigned to all the tested compounds except 13b, 15e and 16b. 
Scheme 4

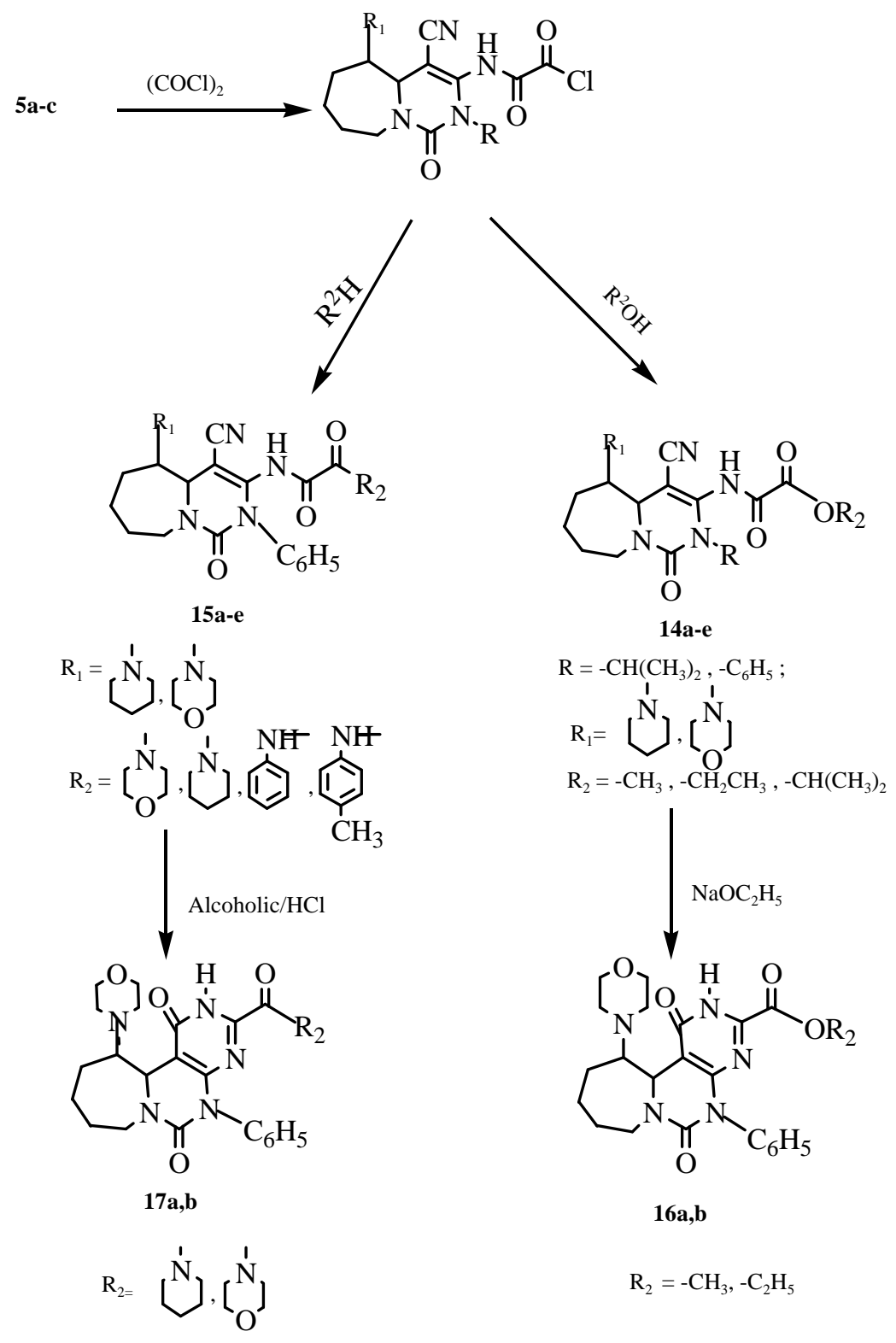




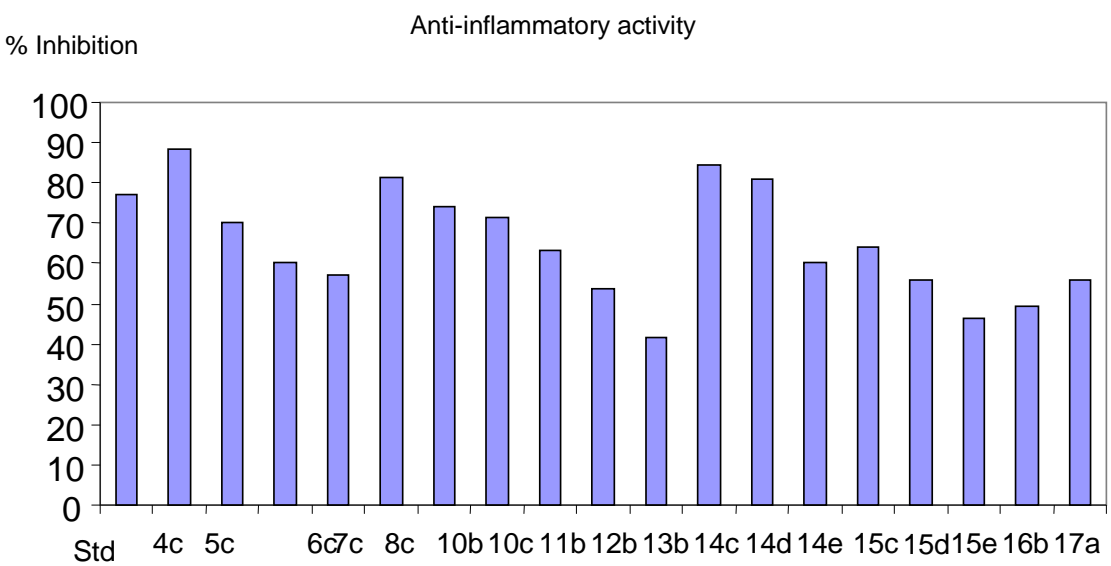

Fig. 1: Graphical representation of the data.

It is noteworthy that compounds 4c, 8c, 14c and 14d displayed potential anti-inflammatory effect as compared to the standard (diclofenacsodium).

Molecular modeling and structureactivity relationship (SAR) of the newly prepared compounds

A comparative measurement of torsion angles of the well known active compounds I, II and III and that of the prepared compounds $\mathbf{4 c}$, 5c, 6c, 7c, 8c, 10b,c, 12b, 13b, 14c,d,e 15c,d,e, 16b, 17a revealed certain 3D structural similarities. A comparison of the electrostatic potentials of these compounds have been constructed and presented. For the sake of molecular modelling study HyperChem software ${ }^{18}$ had been used. The structure models were generated, then fully minimized to obtain the optimum structures using RMS gradient of 0.1 and FletcherReeves algorithm. The electrostatic potential maps were constructed first by calculating the charges by Modified Neglectof Differential Overlap (MNDO) method. The potential maps were calculated derived from a single point charge models at horizontal grid points $=100$ and vertical grid points $=100$ and contour level of 50 and an increment of 0.1 . The obtained isopotential surfaces were moved in space together with the underlying molecules in order to obtain maximum overlap.

Torsion angles of atoms 5-4a-4 $\mathrm{C}(\mathrm{CN}), \quad \mathrm{H} 5-5-4 \mathrm{a}-4, \quad 12-12 \mathrm{a}-12 \mathrm{~b}-\mathrm{CO}$ and $\mathrm{H}(\mathrm{Cl})(\mathrm{N})-12-12 \mathrm{a}-12 \mathrm{~b}$ and the corresponding activity for the chosen compounds are presented in Tables I and II. 
It has been observed that the tested compounds fall into two main categories. The first comprises the bicyclic ring system pyrimido [1,6-a] azepine derivatives while the tricyclic counterparts, the pyrimido[4',5':4,5] pyrimido[1,6-a]azepines, represent the second one. Within the first series, it was observed from the data obtained, that introduction of a morpholine moiety at position 5 of the bicyclic pyrimido[1,6-a]azepine ring system $\mathbf{I}$ resulting in formation of compound $\mathbf{4 c}$ caused significant increase in twisting of torsion angles 5-4a-4-C(CN) and H5-5-4a-4 accompanied with improved activity.

On the other hand, reduction of the imino group at position 3 of compound $\mathbf{4 c}$ resulting in the formation of the corresponding 3amino derivative $\mathbf{5 c}$ led to decreased activity to a large extent probably due to the decrease of twisting of torsion angles as indicated by molecular modeling study.

Different substitution on the amino derivative 5 was done in order to see the influence of these changes on the torsion angles and hence on the activity. When substitution of this amino group was effected with different ester or amide carbonyl group yielding compounds $\mathbf{1 4 c , d , e}$ and compounds $\mathbf{1 5 c}, \mathbf{d}$; no significant difference in the torsion angles was observed. Nevertheless, the difference in activity noted may be due to difference in the electronic factors.
In view of the results obtained for the second series, it was observed that torsion of angle 12-12a-12b(CO) increases upon insertion of chlorine atom at position 12 of the tricycle II leading to great improvement in activity of the product III. The tricycle $\mathbf{6 c}$ bearing morpholine moiety at position 12 (instead of the chlorine atom) retained activity within significant range. Further substitution of the tricycle $\mathbf{6 c}$ at position 3 with different reagents or subjecting it to cyclization was effected in order to see the relevance of these changes on the torsion angles and hence on activity. With substitution no significant difference in torsion angles was perceived accompanied with difference in activity. To our interest, compound $8 \mathbf{c}$ bearing acetic acid moiety at position 3 showed improved activity as compared to the standard.

Additionally, cyclization of the 3chloropropyl derivative $\mathbf{1 2 b}$ resulting in the formulation of the tetracycle 13b led to decreased activity; whilst no significant change in angle 1212a-12b-CO was observed, a slight decrease in twisting of angle $\mathrm{H}(\mathrm{Cl})(\mathrm{N})-12-12 \mathrm{a}-12 \mathrm{~b}$ was noted.

Finally, the bicyclic carbamoyl ester and amide derivatives 14c, 15c revealed higher twisting of torsion angle 5-4a-4-C(CN) and higher activity compared to their cyclized counterparts $\mathbf{1 6 b}, \mathbf{1 7} \mathbf{a}$ confirming our concept. 


\section{D Electrostatic potential maps of some selected compounds}

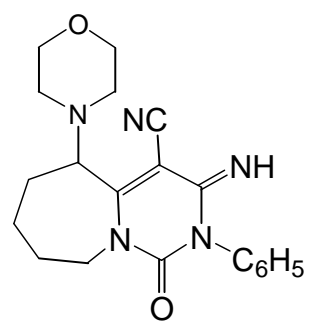

(4c)

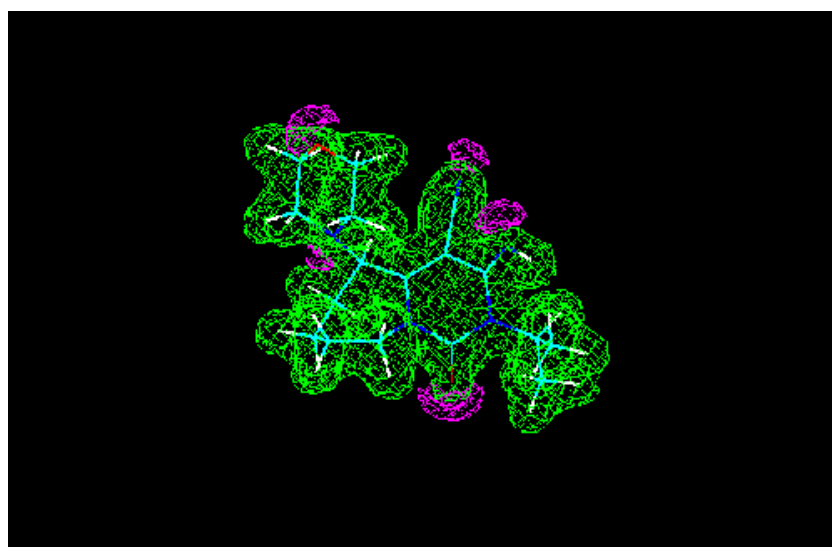

3D Electrostatic potential map of compound (4c) derived from a single point charge model using MNDO charges $-5 \mathrm{kcal} / \mathrm{mol}$ green, $+5 \mathrm{kcal} / \mathrm{mol}$ pink

Torsion angle $\mathrm{C}=-7.98822^{\circ}$

Torsion angle $\mathrm{D}=-88.6645^{\circ}$<smiles>CCCCCN1C(=O)N2CCCCC(N3CCOCC3)C2C(C#N)=C1N</smiles>

(5c)

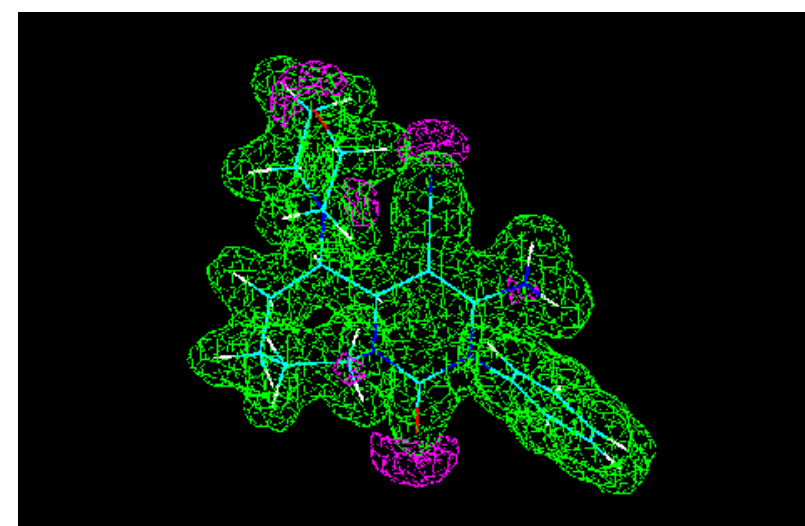

3D Electrostatic potential map of compound (5c) derived from a single point charge model using MNDO charges $-5 \mathrm{kcal} / \mathrm{mol}$ green, $+5 \mathrm{kcal} / \mathrm{mol}$ pink

Torsion angle $\mathrm{C}=3.4387^{\circ}$

Torsion angle $\mathrm{D}=-58.8528^{\circ}$

N.B.: For simplicity letter A will be used to express torsion angle at atom : $12-12 \mathrm{a}-12 \mathrm{~b}-$ $\mathrm{C}(\mathrm{CO})$, letter $\mathrm{B}$ to express the torsion angle at atoms $\mathrm{H}(\mathrm{Cl})(\mathrm{N})-12-12 \mathrm{a}-12 \mathrm{~b}$, letter $\mathrm{C}$ to express torsion angle at atoms $5-4 \mathrm{a}-4-\mathrm{C}(\mathrm{CN})$ and letter $\mathrm{D}$ to express torsion angle at atoms $\mathrm{H}(\mathrm{N})-5-4 \mathrm{a}-4$. 

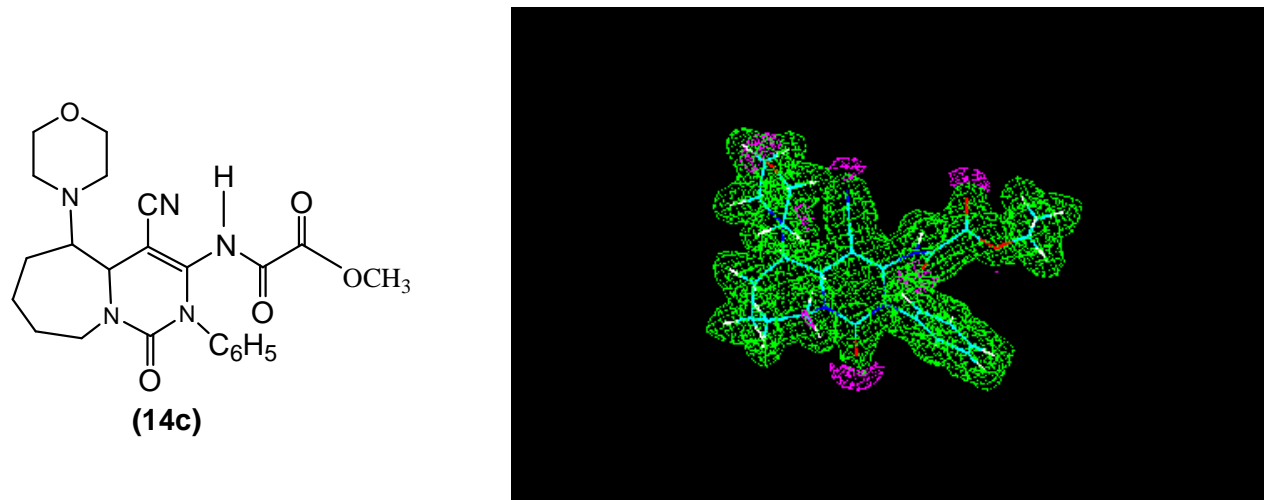

3D Electrostatic potential map of compound (14c) derived from a single point charge model using MNDO charges $-5 \mathrm{kcal} / \mathrm{mol}$ green, $+5 \mathrm{kcal} / \mathrm{mol}$ pink

Torsion angle $\mathrm{C}=2.71576^{\circ}$

Torsion angle $\mathrm{D}=-57.5781^{\circ}$
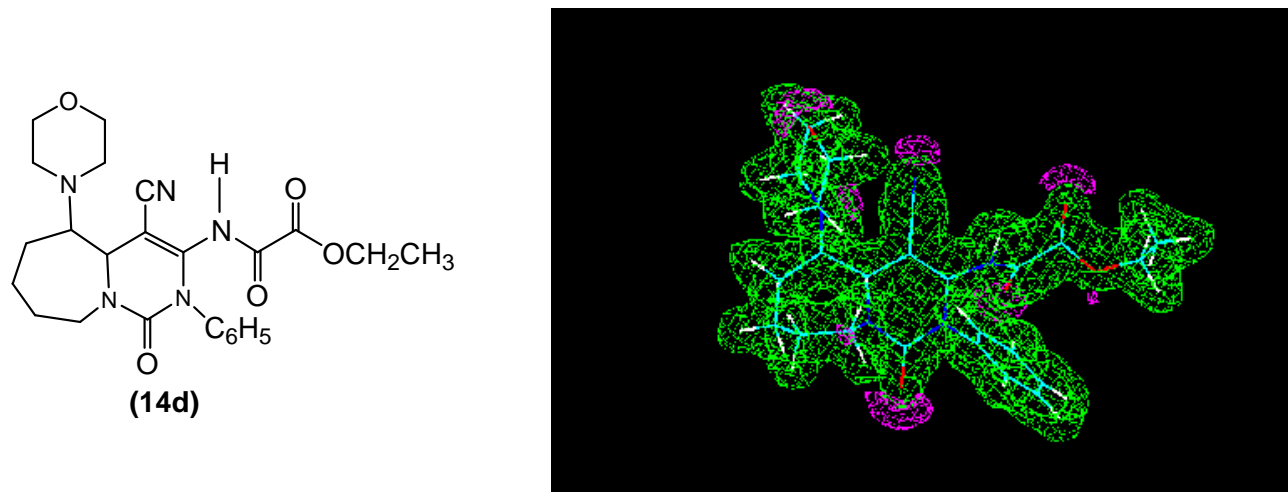

3D Electrostatic potential map of compound (14d) derived from a single point charge model using MNDO charges $-5 \mathrm{kcal} / \mathrm{mol}$ green, $+5 \mathrm{kcal} / \mathrm{mol}$ pink

Torsion angle $\mathrm{C}=3.41576^{\circ}$

Torsion $\mathrm{D}=-56.5781^{\circ}$ 

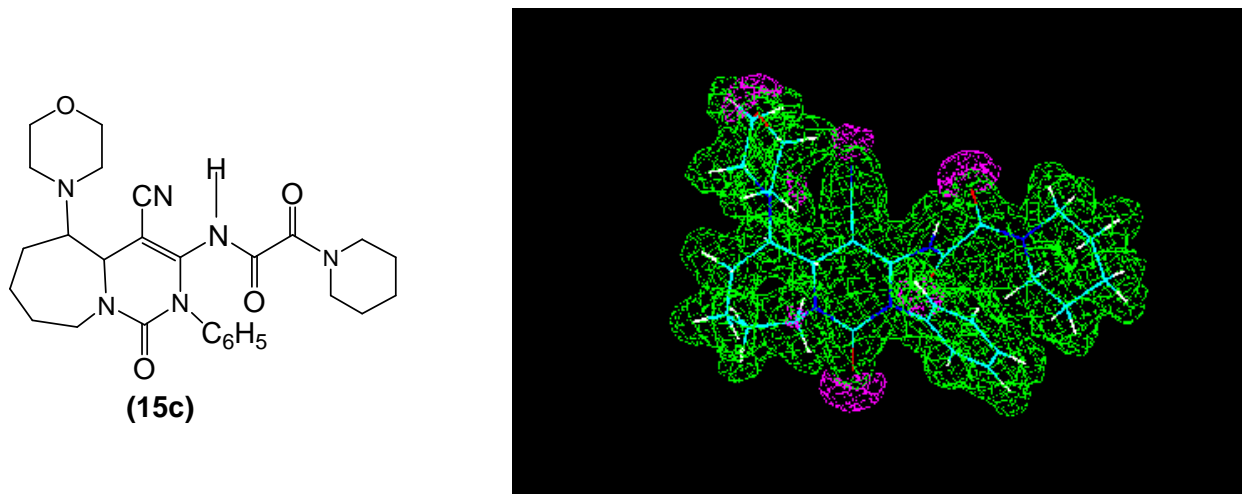

3D Electrostatic potential map of compound $(\mathbf{1 5 c})$ derived from a single point charge model using MNDO charges $-5 \mathrm{kcal} / \mathrm{mol}$ green, $+5 \mathrm{kcal} / \mathrm{mol}$ pink

Torsion angle $\mathrm{C}=5.50074^{\circ}$

Torsion angle $\mathrm{D}=-58.5448^{\circ}$

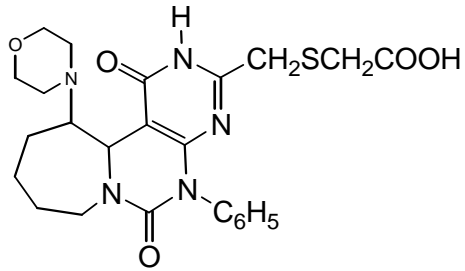

(8c)

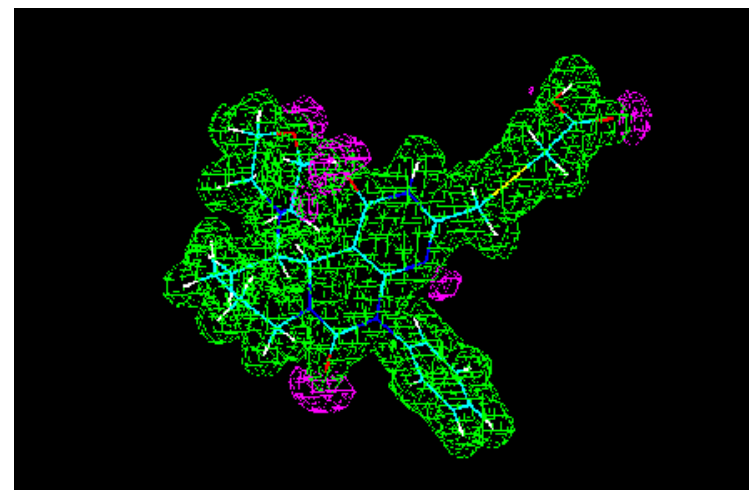

3D Electrostatic potential map of compound $(\mathbf{8 c})$ derived from a single point charge model using MNDO charges $-5 \mathrm{kcal} / \mathrm{mol}$ green, $+5 \mathrm{kcal} / \mathrm{mol}$ pink

Torsion angle $\mathrm{A}=88.9228^{\circ}$

Torsion angle $\mathrm{B}=-43.1943^{\circ}$ 

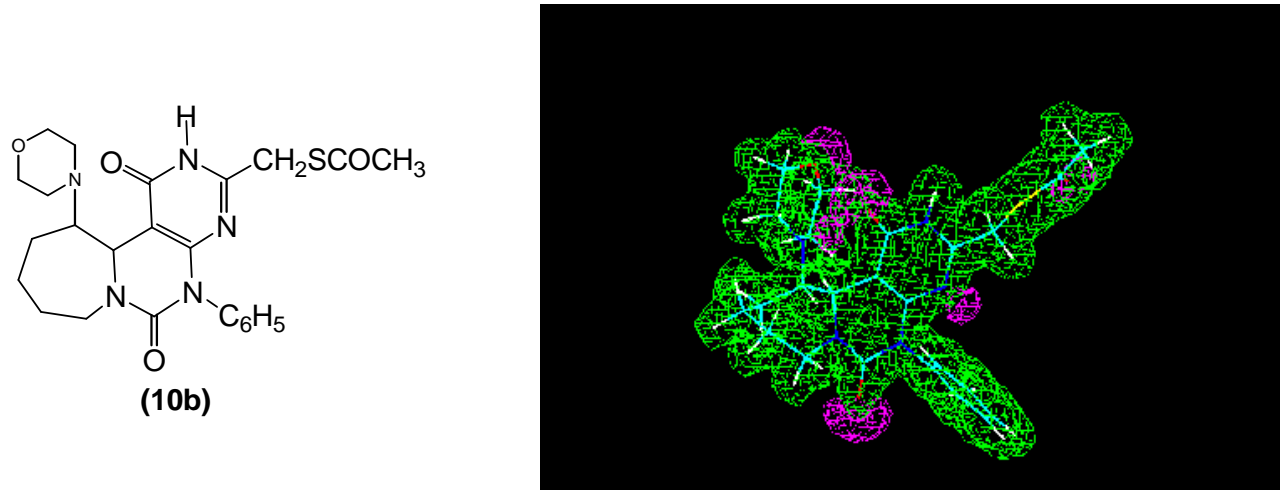

3D Electrostatic potential map of compound (10b) derived from a single point charge model using MNDO charges $-5 \mathrm{kcal} / \mathrm{mol}$ green, $+5 \mathrm{kcal} / \mathrm{mol}$ pink

Torsion angle $\mathrm{A}=89.4528^{\circ}$

Torsion angle $\mathrm{B}=-51.7408$

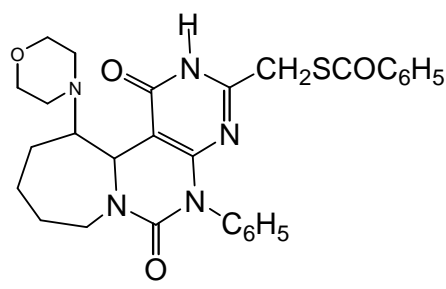

(10c)

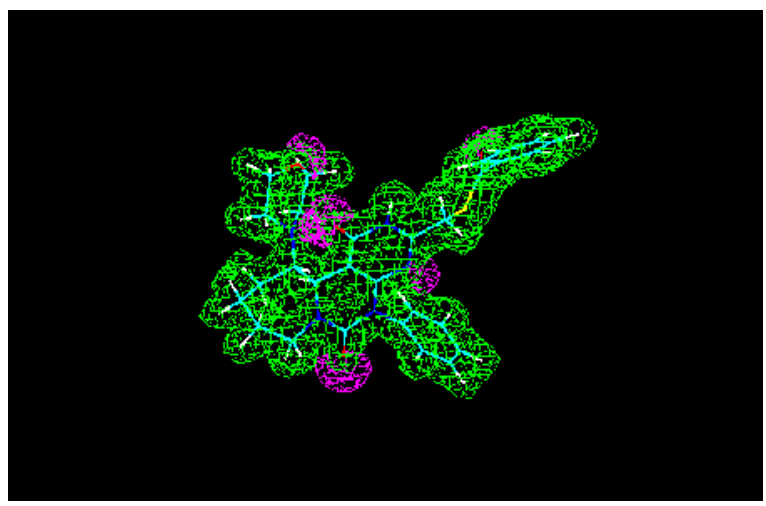

3D Electrostatic potential map of compound (10c) derived from a single point charge model using MNDO charges $-5 \mathrm{kcal} / \mathrm{mol}$ green, $+5 \mathrm{kcal} / \mathrm{mol}$ pink

Torsion angle $\mathrm{A}=89.8137^{\circ}$

Torsion angle $\mathrm{B}=-52.8788^{\circ}$ 


\section{REFERENCES}

1- M.Y. Ebeid, Z. I. Gad, N. M. ElSayed, EL. S. Ahmed and B. ElSayeh, Egypt. J. Pharm. Sci., 32, 441 (1991) (and references therein).

2- M.Y. Ebeid, H. H. Hassanein and N. Obidan, Bull. Fac. Pharm. Cairo, Univ., 33, special issue, 35 (1995).

3- M. Ebeid and I. Bitter, Egypt. J. Pharm. Sci., 19, 349 (1978).

4- C. A. Winter, E. A. Risley, G. W. Nuss, Proc. Soc. Exp. Biol. Med., 111, 544 (1962).

5- P. Schalck and J. Rieker, Angew. Makromol. Chem., 15, 203 (1971).

6- N. M. El-Sayed and M. M. Hussein, Egypt. J. Pharm. Sci., 44, 319 (2003)

7- N. M. El-Sayed and H. A. Abdel Latif, Bull. Fac. Pharm. Cairo, Univ., 31, 55 (1993).

8- N. M. El-Sayed, D. A. Abou El Ella, A. E. Farag, M. Y. Ebeid and N. A. Monieb, ibid., 38, 19 (2000).

9- A. I. Mayers and N. Nazarenko, J. Am. Chem. Soc., 94, 3243 (1972).

10- O. A. Reutov, "Fundamentals of Theoritical Organic Chemistry", $2^{\text {nd }}, \quad$ Scripta Technica Inc., Appleton century Crofts, New York, 1967, p. 216.
11- M. T. Bogert and W. F. Hand, J. Am. Chem. Soc., 24, 1031 (1902).

12- M. Y. Ebeid, N. M. El-Sayed and El-S. Ahmed, "Condensed Pyrimidines XII". Proceedings of XXI Conference of Pharmaceutical Sciences, 1990, pp. 105-112.

13- E. C. Taylor and A. McKillop, "The Chemistry of Cyclic Enaminonitriles and $\mathrm{O}$ Aminonitriles",7, Interscience Publishers, a Division of John Willey and Sons, New York, London, Sydney, Toronto, 1970, p. 227.

14- E. C. Taylor and Y. Shvo, J. Org. Chem., 33, 1719 (1968).

15- A. A. Harb, Egypt. J. Pharm. Sci., 33, 283 (1992).

16- N. M. El-Sayed, M. M. Hanna, N. M. Abdel Gawad, H. H. Hassanein and M. Y. Ebeid, Bull. Fac. Pharm., Cairo Univ., 31, 171 (1993).

17- M. Y. Ebeid, H. H. Hassanein and M. Riad, ibid., 31, 187 (1993).

18- HyperChem TM version 6.03 . Molecular modelling system (2000). 